\title{
On a singular heat equation with dynamic boundary conditions
}

\author{
Giulio Schimperna \\ Dipartimento di Matematica, Università di Pavia, \\ Via Ferrata 1, I-27100 Pavia, Italy \\ E-mail: giusch04@unipv.it \\ Antonio Segatti \\ Dipartimento di Matematica, Università di Pavia, \\ Via Ferrata 1, I-27100 Pavia, Italy \\ E-mail: antonio.segatti@unipv.it \\ Sergey Zelik \\ Department of Mathematics, University of Surrey, \\ Guildford, GU2 7XH, United Kingdom \\ E-mail: S.Zelik@surrey.ac.uk
}

September 27, 2018

\begin{abstract}
In this paper we analyze a singular heat equation of the form $\vartheta_{t}+\Delta \vartheta^{-1}=f$. The singular term $\vartheta^{-1}$ gives rise to very fast diffusion effects. The equation is settled in a smooth bounded domain $\Omega \subset \mathbb{R}^{3}$ and complemented with a general dynamic boundary condition of the form $\alpha \vartheta_{t}-\beta \Delta_{\Gamma} \vartheta=\partial_{\boldsymbol{n}} \vartheta^{-1}$, where $\Delta_{\Gamma}$ is the Laplace-Beltrami operator and $\alpha$ and $\beta$ are nonnegative coefficients (in particular, the homogeneous Neumann case given by $\alpha=\beta=0$ is included). For this problem, we first introduce a suitable weak formulation and prove a related existence result. For more regular initial data, we show that there exists at least one weak solution satisfying instantaneous regularization effects which are uniform with respect to the time variable. In this improved regularity class, uniqueness is also shown to hold.
\end{abstract}

Key words: very fast diffusion, Moser iterations, dynamic boundary conditions.

AMS (MOS) subject classification: 35K55, 35K67, 35K51, 80A22.

\section{Introduction}

In this paper we address a singular heat equation having the expression

$$
\vartheta_{t}+\Delta \vartheta^{-1}=f,
$$

where $f$ is an external force. The equation is settled in a smooth bounded domain $\Omega \subset \mathbb{R}^{3}$, where the restriction to the three-dimensional setting is motivated by physical applications, and is complemented with dynamic boundary conditions of the form

$$
\alpha \vartheta_{t}-\beta \Delta_{\Gamma} \vartheta=\partial_{\boldsymbol{n}} \vartheta^{-1} \quad \text { on } \Gamma:=\partial \Omega,
$$

where $\Delta_{\Gamma}$ is the Laplace-Beltrami operator and $\alpha, \beta \geq 0$ (in particular they may both be 0 , so that the homogeneous Neumann problem is included). Equation (1.1) can be viewed in the framework of nonlinear diffusion problems, i.e., of equations of the form

$$
\vartheta_{t}-\operatorname{div}\left(\vartheta^{m-1} \nabla \vartheta\right)=f,
$$


with $m \in \mathbb{R}$. When $m \geq 1$, equation (1.3) is the well-known porous medium equation [21] (heat equation when $m=1$ ); for $m<1$, equation (1.3) lies in the class of fast diffusion equations (ultra fast when $m<0$ ) analyzed in several papers (see, e.g., the pioneering work [15]). In this regime very fast diffusion occurs in the regions where $\vartheta$ is small. Moreover, one can distinguish two sub-regimes, depending on the so-called first critical fast diffusion exponent $m_{c}:=\frac{d-2}{2}$ ( $d$ is the dimension) which acts as a threshold between the good parameter range $m \in\left(m_{c}, 1\right)$ and its complementary range $m \leq m_{c}$. More precisely, when $m_{c}<m<1$ and the initial condition $\vartheta_{0}$ is non-negative and locally integrable, then equation (1.3) (with $f=0$ ) on the whole space has a unique weak solution which is globally defined, positive and smooth. Moreover, solutions emanating from initial data lying in $L^{p}\left(\mathbb{R}^{d}\right)$ $(p \geq 1)$, or even in the Marcinkiewicz space $M^{p}\left(\mathbb{R}^{d}\right)$, immediately become bounded. The scenario when $m<m_{c}$ is drastically different for at least two reasons (we refer to 4 for further remarks). First of all, solutions may in general be unbounded and may also be non-smooth. As an example, when $\Omega=\mathbb{R}^{3}$, one can consider, for arbitrary $T>0$, the function (see [20])

$$
\Theta(t, x)=2\left((T-t)^{+}|x|^{-2}\right)^{1 / 2},
$$

which solves (1.1) with $f=0$ and initial condition $\Theta_{0}(x)=2 T^{1 / 2}|x|^{-1} \in L_{\text {loc }}^{p}\left(\mathbb{R}^{3}\right)$ for any $p<3$. In particular, $\Theta$ is an unbounded function and $\Theta(t, \cdot) \notin L_{\mathrm{loc}}^{p}\left(\mathbb{R}^{3}\right)$ for $p \geq 3$, at least until it vanishes for $t \geq T$. In this range of $m$, the boundedness of the solutions is tied to the summability of the initial condition and to the value of $m$. More precisely, solutions are bounded whenever $\vartheta_{0} \in L^{p}\left(\mathbb{R}^{d}\right)$ with $p>p_{c}:=\frac{d(1-m)}{2}$ (see [4]). Note that, when $d=3$ and $m=-1$ (as in our case (1.1)), $p_{c}=3$. A second notable feature of the fast diffusion regime, which is already evident in example (1.4), is the possible occurrence of extinction in finite time. This means that a solution may become identically zero after some finite time $T$ which depends on the initial conditions. Consequently, positivity is lost. The ultra fast diffusion regime $m<0$ presents further difficulties linked to the mere question of existence (see 19 and [12). In particular, for (nonzero) data in $L^{p}$ with $p<p_{c}$ one may face a phenomenon called "immediate extinction" meaning that solutions obtained as limits of reasonable approximation schemes can be identically zero for any $t>0$. Note that the immediate extinction can occur also for boundary value problems with zero Dirichlet conditions. Finally, the case $m=m_{*}=\frac{d-4}{d-2}, d \geq 3$, deserves a particular attention, as observed in the papers 2] and [5] dealing with the asymptotics as $t \nearrow T$ (the extinction time). Note that we always have $m_{*}<m_{c}$ and, for $d=3$, we have $m_{*}=-1$, exactly as in our equation (1.1).

In this paper, we are interested in the analysis of equation (1.1) with the dynamic boundary condition (1.2) in the case when the source term $f$ has zero spatial mean. Then, a straightforward computation permits us to see that conservation of mass occurs. For instance, in the case $\alpha=0$, we have $\int_{\Omega} \vartheta(t, x) \mathrm{d} x=\int_{\Omega} \vartheta_{0}(x) \mathrm{d} x$ for any $t>0$, which manifestly excludes both immediate extinction and extinction in finite time. It is worth pointing out that Neumann (or related) boundary problems are poorly studied in the literature, even in the simpler case of the porous medium equation. In this regard, well-posedness and asymptotic results have been established in 1]. More recently, these results have been improved in [14] by means of an approach similar to ours based on Moser iteration techniques.

Our interest is twofold. From the one hand, we aim at proving existence of at least one solution under weak conditions on the initial data. To explain what we mean for "weak", and considering for simplicity the case $f=0$, we note that, thanks to the monotone structure of (1.1)-(1.2), the system admits several Liapunov functionals. In particular, on account of physical considerations (see below for details), we may identify a natural energy functional $\mathcal{E}$, which is defined in (2.12) below. Mathematically, the finiteness of $\mathcal{E}$ seems to be a minimal regularity condition on the initial datum $\vartheta_{0}$ that allows to define a rigorous concept of weak solution (cf. Definition 2.1 below). Indeed (in the simpler Neumann case $\alpha=\beta=0$ ), finiteness of the energy corresponds to asking that $\vartheta_{0}-\log \vartheta_{0} \in L^{1}(\Omega)$. Hence, we have some control on the $L^{1}$-norm of the solution (permitting to use $L^{1}$-arguments in the analysis) and also a positivity condition. Weak solutions emanating from initial data with finite energy $\mathcal{E}$ will be called "energy" solutions. Actually, our first result (see Theorem 5.1 below) states that, if the initial data have finite energy and $f$ has zero mean value and satisfies suitable summability conditions, then at least one global energy solution exists; moreover, the energy $\mathcal{E}(\vartheta)$ remains bounded uniformly in time. 
Once existence is established, we study boundedness and positivity properties of energy solutions. Assuming the (sole) energy regularity of initial data, we can prove (see Theorem 5.1) that $\vartheta(t)$ becomes instantaneously bounded from below, namely, for $t>0$ we have $\vartheta(t) \geq c(t)>0$, where the constant $c(t)$ depends only on the initial energy and may vanish as $t \rightarrow 0$. If, in addition, $\vartheta_{0}$ is (in space dimension $d=3$ ) in some $L^{p}$-space with $p>3\left(=p_{c}\right.$ ), then (see Theorem 5.3 ) we prove an analogous bound from above $(\vartheta(t) \leq C(t)<+\infty$ for $t>0)$. These bounds also entail further regularity properties of the solutions, which hold uniformly for large values of the time variable. Indeed, $c(t)$ does not vanish, and $C(t)$ does not explode, for $t \nearrow \infty$. The main tool in our regularity proof is a suitably modified Moser iteration scheme. Note that the required extra regularity of the initial datum is in complete agreement with the above discussion on the case $\Omega=\mathbb{R}^{d}$ and with the explicit solution (1.4). However, since the choice of dynamic boundary conditions (1.2) precludes the occurrence both of immediate and of finite time extinction, it remains an open challenging question to understand whether the restriction $p>p_{c}$ is still optimal in the present setting. Mathematically, the main difficulties we encountered in the analysis of this problem come from the choice of the boundary conditions (1.2) which does not allow us to perform some otherwise standard a priori estimates. Actually, we have a sort of "asymmetry" of diffusion effects between the equation in the interior domain (where the Laplacian acts on $1 / \vartheta$ ) and that on the boundary (where the Laplace-Beltrami operator acts on (the trace of) $\vartheta$ ). Moreover, as the precise statements of our results show, in some situations we cannot allow $\alpha$, or $\beta$, to be 0 . Note, however, that the situation when both $\alpha$ and $\beta$ vanish is always permitted because it corresponds to the simpler case of no-flux conditions, already studied in the literature.

A natural application of equation (1.1) comes from the so-called phase change models of Penrose-Fife type [16. In this physical context, the unknown $\vartheta$ represents the absolute temperature of a material liable to a phase transition, while the source $f$ can also take into account the effects of the phase variable on the temperature evolution. More precisely, in the Penrose-Fife model, equation (1.1) is coupled with a parabolic equation of Allen-Cahn or Cahn-Hilliard type describing the evolution of the phase variable $\chi$ (see, e.g., [9], 10, [11, 17, [18]). In this framework, the choice of considering a zero-mean-valued forcing function $f$ in (1.1) can be motivated by the need of replicating in our situation some inner cancellation effects that appear in the energy estimates for the full model. An application of the present results to the Penrose-Fife system with boundary conditions of the type (1.2) (which has never been studied in the literature, at least up to our knowledge) will be given in a forthcoming paper. Besides the Penrose-Fife model, equation (1.1) (or, more generally, equation (1.3) with $m<0$ ) comes naturally into play in other physical contexts (see [3] and [8]). For example, it appears [13] in the study of the long-range Van der Waals interactions in thin films that diffuse on a solid surface.

The plan of the paper is as follows. In the next Section 2 we present our assumptions and state a rigorous definition of weak solution. Section 3 is devoted to proving the main technical lemmas constituting the core of our existence proof. In particular, using Moser iteration arguments, we show that, under suitable conditions on data, both $\vartheta$ and $\vartheta^{-1}$ satisfy instantaneous regularization properties. Then, in Section 4 we prove that (global) existence holds for smooth and bounded initial data. Finally, in Section 5 we prove our main results. Namely, we show that existence holds for initial data enjoying the sole "energy" regularity. Moreover, we investigate regularization properties, and (under additional conditions) uniqueness of weak solutions.

\section{Notation and hypotheses}

Let $\Omega$ be a smooth bounded domain of $\mathbb{R}^{3}$ (of course, everything could be easily extended to the one and two dimensional cases, where, actually, better results are expected to hold). Let also $|\Omega|=1$ so that $\|v\|_{L^{p}(\Omega)} \leq\|v\|_{L^{q}(\Omega)}$ for all $1 \leq p \leq q \leq+\infty, v \in L^{q}(\Omega)$. Let $H:=L^{2}(\Omega)$, endowed with the standard scalar product $(\cdot, \cdot)$ and norm $\|\cdot\|$. Let also $V:=H^{1}(\Omega)$. We note by $\|\cdot\|_{X}$ the norm in the generic Banach space $X$. and by $\langle\cdot, \cdot\rangle_{X}$ the duality between $X^{\prime}$ and $X$. We will also write $\|\cdot\|_{p}$ for $\|\cdot\|_{L^{p}(\Omega)}$ and $\|\cdot\|_{p, \Gamma}$ for $\|\cdot\|_{L^{p}(\Gamma)}$, for simplicity. Next, we set $H_{\Gamma}:=L^{2}(\Gamma)$ and $V_{\Gamma}:=H^{1}(\Gamma)$ and denote by $(\cdot, \cdot)_{\Gamma}$ the scalar product in $H_{\Gamma}$, by $\|\cdot\|_{\Gamma}$ the corresponding norm, and by $\langle\cdot, \cdot\rangle_{\Gamma}$ the duality between $V_{\Gamma}^{\prime}$ and $V_{\Gamma}$. We also denote by $\nabla_{\Gamma}$ the tangential gradient on $\Gamma$ and by $\Delta_{\Gamma}$ the 
Laplace-Beltrami operator. We can thus set

$$
\mathcal{H}:=H \times H_{\Gamma} \quad \text { and } \quad \mathcal{V}:=\left\{z \in V:\left.z\right|_{\Gamma} \in V_{\Gamma}\right\} .
$$

The spaces $\mathcal{H}$ and $\mathcal{V}$ are endowed with the natural norms, respectively denoted by $\|\cdot\|_{\mathcal{H}}$ and $\|\cdot\|_{\mathcal{V}}$. For instance, for $h \in \mathcal{H}$, we may set $\|h\|_{\mathcal{H}}^{2}:=\|h\|^{2}+\|h\|_{\Gamma}^{2}$, whereas for $v \in \mathcal{V}$ we put $\|v\|_{\mathcal{V}}^{2}:=$ $\|v\|_{V}^{2}+\left\|\nabla_{\Gamma} v\right\|_{\Gamma}^{2}$. Unless specified otherwise, in the sequel we shall make the following convention: when we write $h \in \mathcal{H}, h$ will be interpreted as a pair of functions belonging, respectively, to $H$ and to $H_{\Gamma}$, and both denoted by the same letter. On the other hand, when we consider $v \in \mathcal{V}$ (or even $v \in V$ ), the symbol $v$ will be intended, depending on the context, either as a function defined on $\Omega$, or as a pair formed by a function of $\Omega$ and its trace on $\Gamma$.

For any function, or functional $z$, defined on $\Omega$, we can then set

$$
m_{\Omega}(z):=\frac{1}{|\Omega|} \int_{\Omega} z=\int_{\Omega} z
$$

where the integral is substituted with the duality $\langle z, 1\rangle$ in case, e.g., $z \in V^{\prime}$. Given $\alpha \geq 0$, we also define the measure $\mathrm{d} m$, given by

$$
\int_{\bar{\Omega}} v \mathrm{~d} m:=\int_{\Omega} v+\alpha \int_{\Gamma} v
$$

where $v$ represents a generic function in $L^{1}(\Omega) \times L^{1}(\Gamma)$. Here and below, integrals over $\Gamma$ are to be intended with respect to the standard surface measure. With some abuse of notation, we will also write

$$
m(v):=\frac{1}{|\Omega|+\alpha|\Gamma|} \int_{\bar{\Omega}} v \mathrm{~d} m=\frac{1}{1+\alpha|\Gamma|} \int_{\bar{\Omega}} v \mathrm{~d} m,
$$

i.e., the "mean value" of $v$ w.r.t. the measure $\mathrm{d} m$. Here $|\Gamma|$ represents the surface measure of $\Gamma$. In case $\alpha=0$, it is intended that $m(v)=m_{\Omega}(v)$. For $p \in[1, \infty)$ and $X$ a Banach space, we introduce the space

$$
\mathcal{T}^{p}(0,+\infty ; X):=\left\{g \in L_{\mathrm{loc}}^{p}(0,+\infty ; X): \sup _{t \in[0,+\infty)}\|g\|_{L^{p}(t, t+1 ; X)}<+\infty\right\}
$$

which is a Banach space as it is endowed with the graph norm. Assuming $f:(0,+\infty) \times \Omega \rightarrow \mathbb{R}$ be a suitable source term and letting $\alpha, \beta \geq 0$, we can introduce the system

$$
\begin{aligned}
& \vartheta_{t}-\Delta u=f, \quad u=-\frac{1}{\vartheta}, \quad \text { in }(0,+\infty) \times \Omega, \\
& \alpha \eta_{t}-\beta \Delta_{\Gamma} \eta=-\partial_{\boldsymbol{n}} u, \quad \text { on }(0,+\infty) \times \Gamma, \\
& \left.\vartheta\right|_{t=0}=\vartheta_{0} \quad \text { in } \Omega, \\
& \left.\alpha \eta\right|_{t=0}=\alpha \eta_{0} \quad \text { on } \Gamma .
\end{aligned}
$$

Our basic assumptions on the initial data are the following:

$$
\begin{aligned}
& \vartheta_{0} \in L^{1}(\Omega), \quad \log \vartheta_{0} \in L^{1}(\Omega), \\
& \alpha \eta_{0} \in L^{1}(\Gamma), \quad \alpha \log \eta_{0} \in L^{1}(\Gamma) .
\end{aligned}
$$

This very natural condition corresponds to asking that the initial data have finite energy, where the energy functional $\mathcal{E}$ is defined as

$$
\mathcal{E}(\vartheta, \eta):=\int_{\Omega}(\vartheta-\log \vartheta)+\alpha \int_{\Gamma}(\eta-\log \eta) .
$$

In the sequel, we will simply write, with some abuse of language, $\mathcal{E}(t)$, in place of $\mathcal{E}(\vartheta(t), \eta(t))$. Moreover, we will note as $\mathbb{E}_{0}$ the energy of initial data, namely

$$
\mathbb{E}_{0}:=\mathcal{E}\left(\vartheta_{0}, \eta_{0}\right) .
$$


The source term is assumed to satisfy for some given $\epsilon \in(0,1)$

$$
f \in L^{2}\left(0,+\infty ; L^{6 / 5}(\Omega)\right) \cap \mathcal{T}^{2}\left(0,+\infty ; L^{3+\epsilon}(\Omega)\right), \quad m_{\Omega}(f)(t)=0, \quad \text { for a.a. } t \in(0,+\infty) .
$$

The first regularity condition seems to be necessary for controlling the energy uniformly in time. The second condition, where we require more summability in space, but allow for a locally uniform (rather than uniform) summability in time, is used for the purpose of Moser iterations. The assumption $m_{\Omega}(f)(t)=0$ is taken because we need a uniform estimate for the spatial mean both of $\vartheta$ and of $u$, which may not hold for general $f$. Actually, integrating (2.6) in $\Omega$, (2.7) on $\Gamma$ and taking the sum, one can see that the "total mass" $m(\vartheta)$ is conserved in time. It is also worth observing that it may be possible to extend our results by considering more general conditions on data (and, particularly, on $f$ ). However we believe that the assumptions provided above are very natural, especially in relation with the energy estimate, and may allow us to give simpler proofs.

In the sequel we will note as Problem $(P)$ the the initial-value problem for system (1.1)-(1.2). In particular, we can introduce a suitable concept of weak solution as follows:

Definition 2.1. A (global) weak solution (or "energy solution") to Problem (P) is a triplet $(\vartheta, \eta, u)$ satisfying, for all $T>0$, the regularity properties

$$
\begin{aligned}
& \vartheta \in C^{0}\left([0, T] ; L^{1}(\Omega)\right), \quad \alpha \eta \in C^{0}\left([0, T] ; L^{1}(\Gamma)\right), \\
& \vartheta>0 \quad \text { a.e. in }(0, T) \times \Omega, \quad \eta>0 \quad \text { a.e. on }(0, T) \times \Gamma, \\
& u \in L^{2}(0, T ; V), \quad u=-1 / \vartheta, \quad \text { a.e. in }(0, T) \times \Omega, \\
& \beta \log \eta \in L^{2}\left(0, T ; V_{\Gamma}\right), \\
& \eta=-1 / u_{\Gamma}, \quad \text { a.e. on }(0, T) \times \Gamma,
\end{aligned}
$$

and fulfilling, for any test function

$$
\xi \in C^{1}\left([0, T] ; C^{0}(\bar{\Omega})\right) \cap C^{0}\left([0, T] ; C^{2}(\bar{\Omega})\right)
$$

and for all times $t \in[0, T]$, the relation

$$
\begin{aligned}
\int_{\Omega} \vartheta(t) \xi(t) & +\alpha \int_{\Gamma} \eta(t) \xi(t)+\int_{0}^{t} \int_{\Omega} \nabla u \cdot \nabla \xi-\beta \int_{0}^{t} \int_{\Gamma} \eta \Delta_{\Gamma} \xi \\
& =\int_{0}^{t} \int_{\Omega} \vartheta \xi_{t}+\alpha \int_{0}^{t} \int_{\Gamma} \eta \xi_{t}+\int_{0}^{t} \int_{\Omega} f \xi+\int_{\Omega} \vartheta_{0} \xi(0)+\alpha \int_{\Gamma} \eta_{0} \xi(0) .
\end{aligned}
$$

Remark 2.2. It is worth giving some explanation of relation (2.19). There, $u_{\Gamma}$ denotes the trace of $u$ on $\Gamma$, which exists for almost every value of the time variable thanks to the first (2.17). More precisely, we have $u_{\Gamma} \in L^{2}\left(0, T ; H^{1 / 2}(\Gamma)\right)$. Actually, for weak solutions we cannot simply write $\eta=\vartheta_{\Gamma}$ since the trace of $\vartheta$ does not necessarily exist. On the other hand, when considering smoother solutions (e.g., in the approximation detailed in Section 4 below) it will happen that $\vartheta$ is more regular so that $\eta$ can be intended, in fact, as the trace of $\vartheta$.

Remark 2.3. In the case when $\alpha=0$ and $\beta>0$, the last integral on the first row of (2.21) may make no sense since (2.15)-(2.19) do not guarantee any summability property for $\eta$, whereas when $\alpha>0$ we can get help from the latter (2.15). For this reason we will be able to consider the case $\alpha=0, \beta>0$ only for more regular solutions (and, correspondingly, under more restrictive conditions on data).

\section{Main technical lemmas}

In this section we prove some regularization estimates holding for sufficiently smooth solutions of Problem (P). In this procedure, a sufficient regularity will always be assumed in such a way that all computations we perform make sense. For this reason, we will indicate simply by $\vartheta$, rather than by $\eta$, the boundary temperature (indeed, the "bulk" $\vartheta$ has a trace at this regularity level). As a rule, we will use the letters $c$ and $\kappa$ to denote generic positive constants, depending only on the set $\Omega$ and on the parameters $\alpha$ and $\beta$, with $\kappa$ used in estimates from below. The dependence, or independence, of $\kappa$ and $c$, with respect to time will be pointed out on occurrence. The values of $c, \kappa$ are allowed to vary from time to time. Finally, $Q$ will denote a generic computable function, increasingly monotone with respect to each of its parameters, taking values in $[0,+\infty)$. 


\subsection{Regularization of $u$}

Lemma 3.1 (Moser iterations for $u$ ). Let $S \in \mathbb{R}, T \geq S+2, \alpha \geq 0, \beta \geq 0$, and let, for some $\epsilon \in(0,1)$,

$$
f \in L^{2}\left(S, T ; L^{3+\epsilon}(\Omega)\right), \quad\|f\|_{L^{2}\left(S, T ; L^{3+\epsilon}(\Omega)\right)}=: F .
$$

Let $(u, \vartheta)$ be a couple of sufficiently smooth functions solving, in a suitable sense, the system

$$
\begin{aligned}
& \vartheta_{t}-\Delta u=f, \quad \vartheta=-1 / u, \quad \text { in } \Omega, \\
& \alpha \vartheta_{t}-\beta \Delta_{\Gamma} \vartheta=-\partial_{n} u, \quad \text { on } \Gamma,
\end{aligned}
$$

over the time interval $(S, T)$. Moreover, let us assume that

$$
\begin{aligned}
& u \in L^{2}(S, T ; V), \quad\|u\|_{L^{2}(S, T ; V)}=: M \\
& u(S) \in L^{1}(\Omega), \quad \alpha u(S) \in L^{1}(\Gamma), \quad\|u(S)\|_{L^{1}(\Omega)}+\alpha\|u(S)\|_{L^{1}(\Gamma)}=: U .
\end{aligned}
$$

Then, for any $\tau \in(0,1)$, we have

$$
\|u\|_{L^{\infty}((S+\tau, T) \times \Omega)} \leq Q\left(F, M, U, \tau^{-1}, T-S\right) .
$$

Proof. We will just consider the case when $\beta=0$, which is more difficult since we cannot get any help from the boundary diffusion term. In this proof, the constant $(\mathrm{s}) c$ are allowed to depend (monotonically) on the length of the time interval $(S, T)$, which is finite and assigned. Indeed, in the sequel this lemma will be applied on time intervals of fixed length. Setting $z:=-u \geq 0$, we will also assume, for the sake of simplicity, that $z \geq 1$ almost everywhere. Indeed, if that does not hold, then it is easy to check that in the estimates below we can simply replace $z$ with $z=\max \{z, 1\}$. That said, we test (3.2) by $z^{p+1}$, where $p \geq 1$ will be specified later. This gives

$$
\frac{1}{p} \frac{\mathrm{d}}{\mathrm{d} t}\left(\|z\|_{p}^{p}+\alpha\|z\|_{p, \Gamma}^{p}\right)+\frac{4(p+1)}{(p+2)^{2}}\left\|\nabla z^{\frac{p+2}{2}}\right\|^{2} \leq \int_{\Omega}|f| z^{p+1} .
$$

In order to recover the full $V$-norm on the left hand side, we can multiply (3.7) by $p$ and then add to both hand sides the quantity

$$
\left\|z^{\frac{p+2}{2}}\right\|_{1}^{2}=\|z\|_{\frac{p+2}{2}}^{p+2}
$$

Hence, using continuity of the embedding $V \subset L^{6}(\Omega)$ and of the trace operator from $V$ to $L^{4}(\Gamma)$, we get on the left hand side of (3.7) a quantity $\mathcal{I}$ such that

$$
\mathcal{I}:=\kappa\left\|\nabla z^{\frac{p+2}{2}}\right\|^{2}+\|z\|_{\frac{p+2}{2}}^{p+2} \geq \kappa\|z\|_{3(p+2)}^{p+2}+\kappa\|z\|_{2(p+2), \Gamma}^{p+2} .
$$

We now choose $p=1$ for the first iteration. Then, we can estimate the right hand side of (3.8) as

$$
\|z\|_{\frac{p+2}{2}}^{p+2} \stackrel{p=1}{=}\|z\|_{\frac{3}{2}}^{3} \leq c\|z\|_{6}^{2}\|z\|_{1} .
$$

On the other hand, still for $p=1$, we can write

$$
\int_{\Omega}|f| z^{2} \leq\|f\|_{3}\left\|z^{1 / 2}\right\|\left\|z^{3 / 2}\right\|_{6} \leq \sigma\|z\|_{9}^{3}+c_{\sigma}\|f\|_{3}^{2}\|z\|_{1},
$$

whence, taking $\sigma$ small enough and collecting (3.8)-(3.11), (3.7) becomes

$$
\frac{\mathrm{d}}{\mathrm{d} t}\left(\|z\|_{1}+\alpha\|z\|_{1, \Gamma}\right)+\kappa\|z\|_{9}^{3}+\kappa\|z\|_{6, \Gamma}^{3} \leq c\left(\|z\|_{6}^{2}+\|f\|_{3}^{2}\right)\|z\|_{1} .
$$

Thus, integrating over $(S, T)$ and using Gronwall's lemma, we readily arrive at

$$
\|z\|_{L^{\infty}\left(S, T ; L^{1}(\Omega)\right)}+\alpha\|z\|_{L^{\infty}\left(S, T ; L^{1}(\Gamma)\right)}+\|z\|_{L^{3}\left(S, T ; L^{9}(\Omega)\right)}+\|z\|_{L^{3}\left(S, T ; L^{6}(\Gamma)\right)} \leq Q(F, M, U),
$$

where the function $Q$ has no explicit dependence on $T$ at this level. This relation is the starting point for the subsequent iterations. 
To proceed, we set $r:=\frac{3+\epsilon}{2+\epsilon}$ to be the conjugate exponent of $3+\epsilon$. Rewriting (3.7) (multiplied by $p$ ) for a suitable new choice of $p$, we can now estimate the right hand side as follows:

$$
p \int_{\Omega}|f| z^{p+1} \leq p\|f\|_{3+\epsilon}\left\|z^{p+1}\right\|_{r}=p\|f\|_{3+\epsilon}\|z\|_{r(p+1)}^{p+1} .
$$

Then, adding again the term $\|z\|_{(p+2) / 2}^{p+2}$ to both hand sides and integrating the result from some $\mathfrak{t} \geq S$ (to be chosen later) to $T$, we arrive at

$$
\begin{aligned}
& \|z\|_{L^{\infty}\left(\mathfrak{t}, T ; L^{p}(\Omega)\right)}^{p}+\alpha\|z\|_{L^{\infty}\left(\mathfrak{t}, T ; L^{p}(\Gamma)\right)}^{p}+\|z\|_{L^{p+2}\left(\mathfrak{t}, T ; L^{3(p+2)}(\Omega)\right)}^{p+2}+\|z\|_{L^{p+2}\left(\mathfrak{t}, T ; L^{2(p+2)}(\Gamma)\right)}^{p+2} \\
& \quad \leq c\|z(\mathfrak{t})\|_{p}^{p}+c \alpha\|z(\mathfrak{t})\|_{p, \Gamma}^{p}+c p F\|z\|_{L^{2(p+1)}\left(\mathfrak{t}, T ; L^{r(p+1)}(\Omega)\right)}^{p+1}+c\|z\|_{L^{p+2}\left(\mathfrak{t}, T ; L^{\frac{p+2}{2}}(\Omega)\right)}^{p+2} \\
& \quad \leq c\|z(\mathfrak{t})\|_{p}^{p}+c \alpha\|z(\mathfrak{t})\|_{p, \Gamma}^{p}+c(p F+1)\|z\|_{L^{2(p+1)\left(\mathfrak{t}, T ; L^{r(p+1)}(\Omega)\right)}}^{p+2},
\end{aligned}
$$

where we used, in particular, that $z \geq 1$. Next, we define, for $i \geq 0$ and $\tau_{i}$ to be chosen later,

$$
J_{i}^{p_{i}}:=\|z\|_{L^{\infty}\left(\tau_{i}, T ; L^{p_{i}}(\Omega)\right)}^{p_{i}}+\|z\|_{L^{p_{i}+2}\left(\tau_{i}, T, L^{3 p_{i}+6}(\Omega)\right)}^{p_{i}+2}+\|z\|_{L^{p_{i}+2}\left(\mathfrak{t}, T ; L^{2\left(p_{i}+2\right)}(\Gamma)\right)}^{p_{i}+2} .
$$

Then, using that $z \geq 1$ and that the integration domain has measure greater than 1 , by elementary interpolation we obtain

$$
\begin{aligned}
\|z\|_{L^{2\left(p_{i+1}+1\right)}\left(\tau_{i}, T ; L^{r\left(p_{i+1}+1\right)}(\Omega)\right)} & \leq\|z\|_{L^{\infty}\left(\tau_{i}, T ; L^{p_{i}}(\Omega)\right)}^{\rho}\|z\|_{L^{p_{i}+2}\left(\tau_{i}, T, L^{3 p_{i}+6}(\Omega)\right)}^{1-\rho} \\
& \leq \rho\|z\|_{L^{\infty}\left(\tau_{i}, T ; L^{p_{i}}(\Omega)\right)}+(1-\rho)\|z\|_{L^{p_{i}+2}\left(\tau_{i}, T, L^{3 p_{i}+6}(\Omega)\right)} \\
& \leq \rho\|z\|_{L^{\infty}\left(\tau_{i}, T ; L^{p_{i}}(\Omega)\right)}+(1-\rho)\|z\|_{L^{p_{i}+2}\left(\tau_{i}, T, L^{3 p_{i}+6}(\Omega)\right)}^{\frac{p_{i}+2}{p_{i}}},
\end{aligned}
$$

where the index $p_{i+1}$ and the interpolation exponent $\rho=\rho(i)$ are given by the system

$$
\left\{\begin{array}{l}
\frac{1-\rho}{p_{i}+2}=\frac{1}{2\left(p_{i+1}+1\right)} \\
\frac{\rho}{p_{i}}+\frac{1-\rho}{3\left(p_{i}+2\right)}=\frac{1}{r\left(p_{i+1}+1\right)}
\end{array}\right.
$$

Dividing the second equation in (3.18) by the first one, we have

$$
\left(\frac{\rho}{p_{i}}+\frac{1-\rho}{3\left(p_{i}+2\right)}\right) \frac{p_{i}+2}{1-\rho}=\frac{2}{r}
$$

whence

$$
\frac{\rho}{p_{i}} \frac{p_{i}+2}{1-\rho}=\frac{2}{r}-\frac{1}{3}=: K_{\epsilon}
$$

and it is easy to compute

$$
K_{\epsilon}=\frac{9+5 \epsilon}{9+3 \epsilon} .
$$

From (3.20) we also infer

$$
\rho=\frac{\frac{p_{i}}{p_{i}+2} K_{\varepsilon}}{1+\frac{p_{i}}{p_{i}+2} K_{\varepsilon}} \in(0,1), \quad \text { provided that } p_{i} \geq 1 .
$$

Being

$$
1-\rho=\frac{1}{1+\frac{p_{i}}{p_{i}+2} K_{\varepsilon}},
$$

we then obtain from the first 3.18

$$
\begin{aligned}
p_{i+1} & =\frac{1}{2} \frac{p_{i}+2}{1-\rho}-1=\frac{1}{2}\left(1+\frac{p_{i}}{p_{i}+2} K_{\varepsilon}\right)\left(p_{i}+2\right)-1 \\
& =\frac{K_{\varepsilon}+1}{2} p_{i}=\frac{9+4 \epsilon}{9+3 \epsilon} p_{i}=: H p_{i},
\end{aligned}
$$


where, obviously, $H=H(\epsilon)>1$ whenever $\epsilon>0$.

Taking the $p_{i}$-th power of (3.17), by convexity we obtain

$$
J_{i} \geq\|z\|_{L^{2\left(p_{i+1}+1\right)}\left(\tau_{i}, T ; L^{r\left(p_{i+1}+1\right)}(\Omega)\right)} .
$$

We can now start the iteration argument. Let $p_{0}=1$ and inductively define, for $i \geq 1, p_{i+1}:=H p_{i}=$ $H^{i+1}$. Moreover, let (for instance), for $i \geq 1$,

$$
\mathfrak{t}_{i}:=\tau \frac{3}{\pi^{2} i^{2}}, \quad \text { so that } \sum_{i=1}^{\infty} \mathfrak{t}_{i}=\frac{\tau}{2}
$$

Let us now rewrite (3.15) by taking $p=p_{i+1}$. Setting also, for brevity, $J_{i}:=J_{p_{i}}$ and writing $\tau_{i+1}$ in place of $\mathfrak{t}$ (to be chosen below), we then obtain, thanks also to (3.25),

$$
J_{i+1}^{p_{i+1}}+\alpha\|z\|_{L^{\infty}\left(\tau_{i+1}, T ; L^{\left.p_{i+1}(\Gamma)\right)}\right.}^{p_{i+1}} \leq c\left\|z\left(\tau_{i+1}\right)\right\|_{p_{i+1}}^{p_{i+1}}+c \alpha\left\|z\left(\tau_{i+1}\right)\right\|_{p_{i+1}, \Gamma}^{p_{i+1}}+c\left(p_{i+1} F+1\right) J_{i}^{p_{i+1}+2} .
$$

Let us now make precise the choice of $\tau_{i}$. First of all, we set $\tau_{0}:=S$. Then, by induction, given $\tau_{i}$, we take $\tau_{i+1} \in\left(\tau_{i}, \tau_{i}+\mathfrak{t}_{i+1}\right)$ such that

$$
\begin{gathered}
\left\|z\left(\tau_{i+1}\right)\right\|_{p_{i+1}}^{p_{i}}+\alpha\left\|z\left(\tau_{i+1}\right)\right\|_{p_{i+1}, \Gamma}^{p_{i}} \leq \frac{1}{\mathfrak{t}_{i+1}} \int_{\tau_{i}}^{\tau_{i}+\mathfrak{t}_{i+1}}\left(\|z(t)\|_{p_{i+1}}^{p_{i}}+\alpha\|z(t)\|_{p_{i+1}, \Gamma}^{p_{i}}\right) \mathrm{d} t \\
\leq c \frac{i^{2}}{\tau} \int_{\tau_{i}}^{\tau_{i}+\mathfrak{t}_{i+1}}\left(\|z(t)\|_{3\left(p_{i}+2\right)}^{p_{i}}+\alpha\|z(t)\|_{2\left(p_{i}+2\right), \Gamma}^{p_{i}}\right) \mathrm{d} t \leq c \frac{i^{2}}{\tau} J_{i}^{p_{i}},
\end{gathered}
$$

where we used that $p_{i+1} \leq 2\left(p_{i}+2\right)$. Thus, (3.27)-(3.28) give

$$
J_{i+1}^{p_{i+1}}+\alpha\|z\|_{L^{\infty}\left(\tau_{i+1}, T ; L^{\left.p_{i+1}(\Gamma)\right)}\right.}^{p_{i+1}} \leq c i^{2 H} \tau^{-H} J_{i}^{p_{i+1}}+c\left(p_{i+1} F+1\right) J_{i}^{p_{i+1}+2} .
$$

In particular, we obtain

$$
J_{i+1}^{H^{i+1}} \leq c\left(i^{2 H} \tau^{-H}+H^{i+1} F+1\right) J_{i}^{H^{i+1}+2} .
$$

Thus, setting $\eta_{i}:=\left(H^{i}+2\right) / H^{i}$, we have

$$
J_{i+1} \leq B_{i}^{H^{-(i+1)}} J_{i}^{\eta_{i+1}}, \quad \text { where } B_{i}:=c\left(i^{2 H} \tau^{-H}+H^{i+1} F+1\right) .
$$

Hence, letting $L_{i}:=\log J_{i}$ and $\zeta_{i}:=\log B_{i}$, we can rewrite (3.31), for $i$ large enough, as

$$
\begin{aligned}
L_{i+1} & \leq H^{-(i+1)} \zeta_{i}+\eta_{i+1} L_{i} \leq H^{-(i+1)} \zeta_{i}+\eta_{i+1}\left(H^{-i} \zeta_{i-1}+\eta_{i} L_{i-1}\right) \\
& \leq H^{-(i+1)} \zeta_{i}+\eta_{i+1} H^{-i} \zeta_{i-1}+\eta_{i+1} \eta_{i}\left(H^{-(i-1)} \zeta_{i-2}+\eta_{i-1} L_{i-2}\right) \\
& \leq \cdots \leq L_{0} \prod_{k=1}^{i+1} \eta_{k}+\sum_{k=1}^{i+1}\left(\zeta_{k-1} H^{-k} \prod_{j=k+1}^{i+1} \eta_{j}\right)
\end{aligned}
$$

where the last productory is understood to be 1 for $k=i+1$. Then, letting $i \nearrow \infty$, it is not difficult to obtain (3.6), which concludes the proof (see also [17] for more details).

If $u$ is bounded at the initial time, we can avoid all complications connected with the choice of the sequence $\mathfrak{t}_{i}$. Indeed, a straightforward modification of the above proof permits to show the following

Corollary 3.2. Let $S \in \mathbb{R}, T \geq S+2, \alpha \geq 0, \beta \geq 0$, and let, for some $\epsilon \in(0,1)$, (3.1) hold. Let, as before, $(u, \vartheta)$ be a couple of sufficiently smooth functions solving, in a suitable sense, system (3.2) -(3.3) over the time interval $(S, T)$. Moreover, let us assume (3.4) together with

$$
u(S) \in L^{\infty}(\Omega), \quad \alpha u(S) \in L^{\infty}(\Gamma), \quad\|u(S)\|_{L^{\infty}(\Omega)}+\alpha\|u(S)\|_{L^{\infty}(\Gamma)}=: U .
$$

Then, we have

$$
\|u\|_{L^{\infty}((S, T) \times \Omega)} \leq Q(F, M, U, T-S) .
$$




\subsection{Regularization of $\vartheta$}

Lemma 3.3 (uniform stability of $L^{p}$ norm). Let $S \in \mathbb{R}, T \geq S+2, \alpha \geq 0, \beta \geq 0$. Let $(u, \vartheta)$ be a couple of sufficiently smooth functions solving in a suitable sense system (3.2)-(3.3) over $(S, T)$. Let also the following properties hold:

$$
\begin{aligned}
& f \in L^{2}\left(S, T ; L^{3}(\Omega)\right), \quad\|f\|_{L^{2}\left(S, T ; L^{3}(\Omega)\right)}=: F \\
& \vartheta \in L^{\infty}\left(S, T ; L^{1}(\Omega)\right), \quad\|\vartheta\|_{L^{\infty}\left(S, T ; L^{1}(\Omega)\right)}=: L, \\
& \vartheta(S) \in L^{p}(\Omega), \quad \alpha \vartheta(S) \in L^{p}(\Gamma), \quad\|\vartheta(S)\|_{L^{p}(\Omega)}+\alpha\|\vartheta(S)\|_{L^{p}(\Gamma)}=: \Theta,
\end{aligned}
$$

where the exponent $p$ is given and is assumed to satisfy $p \in[4, \infty)$ in the case when $\alpha>0$ and $\beta=0$, and is assumed to satisfy $p \in[3, \infty)$ in all the other cases. Then,

$$
\|\vartheta\|_{L^{\infty}\left(S, T ; L^{p}(\Omega)\right)}+\alpha\|\vartheta\|_{L^{\infty}\left(S, T ; L^{p}(\Gamma)\right)} \leq Q(L, F, \Theta),
$$

where the expression of $Q$ is independent of the final time $T$.

Proof. Let us consider first the case when $p \leq 4$. We test (3.2) by $\vartheta^{p-1}$. This gives

$$
\frac{\mathrm{d}}{\mathrm{d} t}\left(\|\vartheta\|_{p}^{p}+\alpha\|\vartheta\|_{p, \Gamma}^{p}\right)+\frac{4 p(p-1)}{(p-2)^{2}}\left\|\nabla \vartheta^{\frac{p-2}{2}}\right\|^{2}+\beta \frac{4 p(p-1)}{p^{2}}\left\|\nabla_{\Gamma} \vartheta^{\frac{p}{2}}\right\|_{\Gamma}^{2} \leq p \int_{\Omega}|f| \vartheta^{p-1} .
$$

Then, being $p \leq 4$, using (3.36), we can add the inequality

$$
\left\|\vartheta^{\frac{p-2}{2}}\right\|_{1}^{2}+\beta\|\vartheta\|_{1}^{p} \leq Q(L) .
$$

Then, owing also to the continuity of the trace operator from $V$ to $L^{4}(\Gamma)$, we may conclude that

$$
\begin{aligned}
& \frac{4 p(p-1)}{(p-2)^{2}}\left\|\nabla \vartheta^{\frac{p-2}{2}}\right\|^{2}+\left\|\vartheta^{\frac{p-2}{2}}\right\|_{1}^{2} \geq \kappa\|\vartheta\|_{3(p-2)}^{p-2}+\kappa\|\vartheta\|_{2(p-2), \Gamma}^{p-2}, \\
& \beta \frac{4 p(p-1)}{p^{2}}\left\|\nabla_{\Gamma} \vartheta^{\frac{p}{2}}\right\|_{\Gamma}^{2}+\beta\|\vartheta\|_{1}^{p} \geq \kappa \beta\|\vartheta\|_{p, \Gamma}^{p} .
\end{aligned}
$$

Here and below, the constants $c$ and $\kappa$ are assumed to be independent of $p$ and of time. Next, we estimate the right hand side of (3.39) as follows:

$$
p \int_{\Omega}|f| \vartheta^{p-1} \leq c\|f\|_{3}\left\|\vartheta^{\frac{p}{2}}\right\|_{2}\left\|\vartheta^{\frac{p-2}{2}}\right\|_{6} \leq \sigma\|\vartheta\|_{3(p-2)}^{p-2}+c_{\sigma}\|f\|_{3}^{2}\|\vartheta\|_{p}^{p}
$$

Taking $\sigma$ small enough, we then arrive at the inequality

$$
\frac{\mathrm{d}}{\mathrm{d} t}\left(\|\vartheta\|_{p}^{p}+\alpha\|\vartheta\|_{p, \Gamma}^{p}\right)+\kappa\|\vartheta\|_{3(p-2)}^{p-2}+\kappa\|\vartheta\|_{2(p-2), \Gamma}^{p-2}+\kappa \beta\|\vartheta\|_{p, \Gamma}^{p} \leq c\|f\|_{3}^{2}\|\vartheta\|_{p}^{p}+Q(L) .
$$

Let us now set

$$
y(t):=\left(\|\vartheta\|_{p}^{p}+\alpha\|\vartheta\|_{p, \Gamma}^{p}\right)^{1 / p} .
$$

Then, we need to distinguish between two different cases. First, let either $\alpha=0$ or both $\alpha$ and $\beta$ be strictly positive. Then, thanks to the assumption $p \geq 3$, it follows that $3(p-2) \geq p$. Moreover, assuming for simplicity $\vartheta \geq 1$, which is of course not restrictive, (3.44) reduces to the differential inequality

$$
\frac{\mathrm{d}}{\mathrm{d} t} y^{p}(t)+\kappa y^{p-2}(t) \leq c\|f(t)\|_{3}^{2} y^{p}(t)+Q(L) .
$$

Then, integrating explicitly the ODE and applying the comparison principle, it is not difficult to obtain (3.38). We observe in particular that the procedure works on time intervals of any length with no explicit dependence of $Q$ on time. In other words, the resulting function $Q$ depends on $T$ only through the quantities $F$ and $L$. Note also that the dissipative term $\kappa y^{p-2}(t)$ on the left hand side is essential for the purpose of getting rid of the quantity $Q(L)$ on time intervals of arbitrary length. 
Second, let us consider the case when $\alpha>0$ and $\beta=0$. Then, we need to use the boundary term resulting from (3.41). This is what forces us to assume $p \geq 4$ so that $2(p-2) \geq p$ (hence, in fact, we are taking exactly $p=4$ at least as a first iteration). Under these assumptions, we still deduce a differential inequality of the form (3.46), which is integrated exactly as before.

Finally, let us remove the restriction $p \leq 4$ made at the beginning. In that case, we need to iterate the procedure a finite number of times. Namely, we can first take $p=4$ to get an estimate of $\vartheta$ in $L^{\infty}\left(S, T ; L^{4}(\Omega)\right.$ ) (and of $\alpha \vartheta$ in $L^{\infty}\left(S, T ; L^{4}(\Gamma)\right)$ ). Thanks to this new information, we can repeat the procedure by taking higher values of $p$. We omit the details and just point out that a similar argument will be carried out in the Lemmas 3.4, 3.5 below.

Lemma 3.4 (Moser iterations for $\vartheta$ ). Let $S \in \mathbb{R}, T \geq S+2, \alpha \geq 0, \beta \geq 0$. Moreover, if $\beta=0$ let also $\alpha=0$. Let $(u, \vartheta)$ be a couple of sufficiently smooth functions solving in a suitable sense system (3.2)-(3.3) over $(S, T)$. Moreover, let us assume that, for some $\epsilon \in(0,1)$,

$$
\begin{aligned}
& f \in L^{2}\left(S, T ; L^{3+\epsilon}(\Omega)\right), \quad\|f\|_{L^{2}\left(S, T ; L^{3+\epsilon}(\Omega)\right)}=: F, \\
& \vartheta \in L^{\infty}\left(S, T ; L^{1}(\Omega)\right), \quad\|\vartheta\|_{L^{\infty}\left(S, T ; L^{1}(\Omega)\right)}=: L, \\
& \vartheta(S) \in L^{3+\epsilon}(\Omega), \quad \alpha \vartheta(S) \in L^{3+\epsilon}(\Gamma), \quad\|\vartheta(S)\|_{L^{3+\epsilon}(\Omega)}+\alpha\|\vartheta(S)\|_{L^{3+\epsilon}(\Gamma)}=: \Theta .
\end{aligned}
$$

Then, for any $\tau \in(0,1)$, we have

$$
\|\vartheta\|_{L^{\infty}((S+\tau, T) \times \Omega)}+\alpha\|\vartheta\|_{L^{\infty}((S+\tau, T) \times \Gamma)} \leq Q\left(F, L, \Theta, \tau^{-1}, T-S\right) .
$$

Proof. In this proof, we work on time intervals of assigned finite length. Hence, we may assume, as in Lemma 3.1 explicit dependence of $c$ on the length $T-S$. To start with a further Moser iteration procedure, we rewrite (3.39), multiply it by $p$, and then add to both sides the term $\left\|\vartheta^{\frac{p-2}{2}}\right\|_{1}^{2}$, which may be controlled as in (3.40). We then deduce

$$
\frac{\mathrm{d}}{\mathrm{d} t}\left(\|\vartheta\|_{p}^{p}+\alpha\|\vartheta\|_{p, \Gamma}^{p}\right)+\kappa\|\vartheta\|_{3(p-2)}^{p-2}+\beta \kappa\|\vartheta\|_{3(p-2), \Gamma}^{p-2} \leq p \int_{\Omega}|f| \vartheta^{p-1}+c\|\vartheta\|_{\frac{p-2}{2}}^{p-2} .
$$

Then, in the case $\alpha=\beta=0$, the proof works similarly with [18, Lemma 3.5], to which we refer the reader for details. In the cases $\alpha, \beta>0$ and $\alpha=0, \beta>0$, the argument can be adapted just with small variants. Note in particular that, at the first iteration step, we choose $p=p_{0}=3+\epsilon$ so that the latter term in (3.51) can be controlled by using Lemma 3.3.

In the case when $\beta=0$ and $\alpha>0$, we can still prove regularization of $\vartheta$, but the argument is a bit more delicate since, as in the corresponding case in Lemma 3.3. we cannot take advantage of the boundary gradient term. So, we have to use the trace theorem as in Lemma 3.1 As before, this forces us to assume some more summability of the initial value of $\vartheta$. Namely, we have

Lemma 3.5 (Moser iterations for $\vartheta$, case $\alpha>0, \beta=0$ ). Let $S \in \mathbb{R}, T \geq S+2, \alpha>0$, and $\beta=0$. Let $(u, \vartheta)$ be a couple of sufficiently smooth functions solving in a suitable sense system (3.2)-(3.3) over $(S, T)$. Moreover, let us assume that, for some $\epsilon \in(0,1)$, (3.47)-(3.48) hold, together with

$$
\vartheta(S) \in L^{4+\epsilon}(\Omega), \quad \vartheta(S) \in L^{4+\epsilon}(\Gamma), \quad\|\vartheta(S)\|_{L^{4+\epsilon}(\Omega)}+\|\vartheta(S)\|_{L^{4+\epsilon}(\Gamma)}=: \Theta .
$$

Then, for any $\tau \in(0, \min \{1, T-S\})$, (3.50) holds.

Proof. As before, here we allow $c$ to depend on $T-S$. We take for simplicity $\alpha=1$. The analogue of (3.51) reads now

$$
\frac{\mathrm{d}}{\mathrm{d} t}\left(\|\vartheta\|_{p}^{p}+\|\vartheta\|_{p, \Gamma}^{p}\right)+\kappa\left\|\vartheta^{\frac{p-2}{2}}\right\|_{V}^{2} \leq p \int_{\Omega}|f| \vartheta^{p-1}+c\|\vartheta\|_{\frac{p-2}{2}}^{p-2} .
$$

Then, we take first $p=4+\epsilon$. Owing to Lemma 3.3 we then get

$$
\|\vartheta\|_{L^{\infty}\left(S, T ; L^{4+\epsilon}(\Omega)\right)}+\|\vartheta\|_{L^{\infty}\left(S, T ; L^{4+\epsilon}(\Gamma)\right)} \leq Q(F, L, \Theta) .
$$


Let us now repeat (3.53) for a new $p$ to be chosen below. Let also $r=\left(3+\epsilon^{\prime}\right) /\left(2+\epsilon^{\prime}\right)$, where $\epsilon^{\prime}$ is a number, also to be chosen below, in the range $(0, \epsilon]$. We then obtain

$$
p \int_{\Omega}|f| \vartheta^{p-1} \leq p\|f\|_{3+\epsilon^{\prime}}\left\|\vartheta^{p-1}\right\|_{r} \leq p\|f\|_{3+\epsilon}\|\vartheta\|_{r(p-1)}^{p-1}
$$

Hence, integrating (3.53) over $(\mathfrak{t}, T)$, for some $\mathfrak{t} \geq S$ (to be chosen later) and using once more continuity of the trace from $V$ to $L^{4}(\Gamma)$, we arrive (compare with (3.15)) at

$$
\begin{aligned}
& \|\vartheta\|_{L^{\infty}\left(\mathfrak{t}, T ; L^{p}(\Omega)\right)}^{p}+\|\vartheta\|_{L^{\infty}\left(\mathfrak{t}, T ; L^{p}(\Gamma)\right)}^{p}+\|\vartheta\|_{L^{p-2}\left(\mathfrak{t}, T ; L^{2 p-4}(\Gamma)\right)}^{p-2}+\|\vartheta\|_{L^{p-2}\left(\mathfrak{t}, T ; L^{3 p-6}(\Omega)\right)}^{p-2} \\
& \leq\|\vartheta(\mathfrak{t})\|_{p}^{p}+\|\vartheta(\mathfrak{t})\|_{p, \Gamma}^{p}+c p F\|\vartheta\|_{L^{2(p-1)}\left(\mathfrak{t}, T ; L^{r(p-1)}(\Omega)\right)}^{p-1}+c\|\vartheta\|_{L^{p-2}\left(\mathfrak{t}, T ; L^{\frac{p-2}{2}}(\Omega)\right)}^{p-2} \\
& \leq\|\vartheta(\mathfrak{t})\|_{p}^{p}+\|\vartheta(\mathfrak{t})\|_{p, \Gamma}^{p}+c(p F+1)\|\vartheta\|_{L^{2(p-1)}\left(\mathfrak{t}, T ; L^{r(p-1)}(\Omega)\right)}^{p-1}
\end{aligned}
$$

Now, we perform Moser iterations as in the proof of Lemma 3.1 starting from $p_{0}=4+\epsilon$. Then, in place of (3.18), we get the system

$$
\left\{\begin{array}{l}
\frac{1-\rho}{p_{i}-2}=\frac{1}{2\left(p_{i+1}-1\right)} \\
\frac{\rho}{p_{i}}+\frac{1-\rho}{3\left(p_{i}-2\right)}=\frac{1}{r\left(p_{i+1}-1\right)}
\end{array}\right.
$$

whence one computes, similarly as before,

$$
K_{\epsilon^{\prime}}=\frac{9+5 \epsilon^{\prime}}{9+3 \epsilon^{\prime}}
$$

and, finally,

$$
\begin{aligned}
p_{i+1} & =\frac{1}{2} \frac{p_{i}-2}{1-\rho}+1=\frac{1}{2}\left(1+\frac{p_{i}}{p_{i}-2} K_{\epsilon^{\prime}}\right)\left(p_{i}-2\right)+1 \\
& =\frac{K_{\epsilon^{\prime}}+1}{2} p_{i}=\frac{9+4 \epsilon^{\prime}}{9+3 \epsilon^{\prime}} p_{i}=: H p_{i}=H^{i+1} p_{0} .
\end{aligned}
$$

Note that, as before, $H$ is larger than 1 since $\epsilon^{\prime}>0$.

Then, we can follow with minor variations the proof of Lemma 3.1 up to formula (3.27). In particular, $J_{i}$ is now defined by setting

$$
J_{i}^{p_{i}}:=\|\vartheta\|_{L^{\infty}\left(\tau_{i}, T ; L^{p_{i}}(\Omega)\right)}^{p_{i}}+\|\vartheta\|_{L^{\infty}\left(\tau_{i}, T ; L^{\left.p_{i}(\Gamma)\right)}\right.}^{p_{i}}+\|\vartheta\|_{L^{p_{i}-2}\left(\tau_{i}, T ; L^{2 p_{i}-4}(\Gamma)\right)}^{p_{i}-2}+\|\vartheta\|_{L^{p_{i}-2}\left(\tau_{i}, T ; L^{3 p_{i}-6}(\Omega)\right)}^{p_{i}-2} .
$$

Then, the analogue of (3.28) reads

$$
\begin{gathered}
\left\|\vartheta\left(\tau_{i+1}\right)\right\|_{p_{i+1}}^{p_{i}-2}+\left\|\vartheta\left(\tau_{i+1}\right)\right\|_{p_{i+1}, \Gamma}^{p_{i}-2} \leq \frac{1}{\mathfrak{t}_{i+1}} \int_{\tau_{i}}^{\tau_{i}+\mathfrak{t}_{i+1}}\left(\|\vartheta(t)\|_{p_{i+1}}^{p_{i}-2}+\|\vartheta(t)\|_{p_{i+1}, \Gamma}^{p_{i}-2}\right) \mathrm{d} t \\
\leq c \frac{i^{2}}{\tau} \int_{\tau_{i}}^{\tau_{i}+\mathfrak{t}_{i+1}}\left(\|\vartheta(t)\|_{3\left(p_{i}-2\right)}^{p_{i}-2}+\|\vartheta(t)\|_{2\left(p_{i}-2\right), \Gamma}^{p_{i}-2}\right) \mathrm{d} t \leq c \frac{i^{2}}{\tau} J_{i}^{p_{i}} .
\end{gathered}
$$

Hence, to control the right hand side of (3.56), we also need to take the $\frac{p_{i}}{p_{i}-2}$-th power of the above inequality. It is readily checked that this does not affect the validity of the argument since the exponent $\frac{p_{i}}{p_{i}-2}$ is asymptotically close to 1 . Note also that (3.61) holds provided that $p_{i+1} \leq 2\left(p_{i}-2\right)$. As $p_{i+1}$ is given by (3.59), this means that we need

$$
(4+\epsilon) H^{i+1} \leq 2\left((4+\epsilon) H^{i}-2\right) .
$$

which is easily shown to be true for every $i \geq 0$ provided that we choose $\epsilon^{\prime} \in(0, \epsilon]$ so small that

$$
(4+\epsilon) H=(4+\epsilon) \frac{9+4 \epsilon^{\prime}}{9+3 \epsilon^{\prime}} \leq 2((4+\epsilon)-2) \text {. }
$$

Actually, also the choice $\epsilon^{\prime}=\epsilon$ works. Notice that, since we used $p_{i}<p_{i+1} \leq 2\left(p_{i}-2\right)$, it follows that the regularity (3.52) is needed in order for the iteration scheme to work. From this point on, the proof proceeds once again similarly with that of Lemma 3.1, hence we can omit the details. 
As before, we have a better result in case the "initial" value of $\vartheta$ is uniformly bounded. The proof follows the preceding ones up to straightforward modifications.

Corollary 3.6. Let $S \in \mathbb{R}, T \geq S+2, \alpha \geq 0, \beta \geq 0$. Let $(u, \vartheta)$ be a couple of sufficiently smooth functions solving in a suitable sense system (3.2)-(3.3) over (S,T). Let also (3.47)-(3.48) hold, together with

$$
\vartheta(S) \in L^{\infty}(\Omega), \quad \alpha \vartheta(S) \in L^{\infty}(\Gamma), \quad\|\vartheta(S)\|_{L^{\infty}(\Omega)}+\alpha\|\vartheta(S)\|_{L^{\infty}(\Gamma)}=: \Theta .
$$

Then, we have

$$
\|\vartheta\|_{L^{\infty}((S, T) \times \Omega)} \leq Q(L, F, \Theta, T-S) .
$$

Remark 3.7. The $L^{3+\epsilon}$ space regularity of $f$, stated in (2.14) with $\epsilon>0$, is strongly exploited in the proofs (cf. (3.24) and (3.58)). Indeed, if one weakens it by putting $\epsilon=0$, no additional summability is gained at subsequent iteration steps and the argument fails.

\subsection{Further regularity of time derivatives}

We now prove that, for smooth solutions of Problem (P), the boundedness of $\vartheta$ and $u$ implies some $L^{p}$-regularity of time derivatives at least for strictly positive times. The proof of this result is a little bit tricky in the case $\beta>0$ since the diffusion operators on $\Omega$ and on $\Gamma$ act on different functions. In particular, we cannot treat the case when $\beta>0$ and $\alpha=0$ because in the derivation of the estimates the contribution of the boundary term $\vartheta_{t}$ is explicitly needed.

Lemma 3.8. Let $S \in \mathbb{R}, T>S, \alpha \geq 0, \beta \geq 0$. If $\beta>0$, then let $\alpha>0$. Let $(u, \vartheta)$ be a couple of sufficiently smooth functions solving in a suitable sense system (3.2)-(3.3) over the time interval $(S, T)$. Moreover, let

$$
\begin{aligned}
& u \in L^{2}(S, T ; V), \quad \beta \vartheta \in L^{2}\left(S, T ; V_{\Gamma}\right), \quad\|u\|_{L^{2}(S, T ; V)}+\beta\|\vartheta\|_{L^{2}\left(S, T ; V_{\Gamma}\right)} \leq U, \\
& f \in L^{2}(S, T ; H), \quad\|f\|_{L^{2}(S, T ; H)}=: F, \\
& u, \vartheta \in L^{\infty}((S, T) \times \Omega), \quad\|u\|_{L^{\infty}((S, T) \times \Omega)}+\|\vartheta\|_{L^{\infty}((S, T) \times \Omega)} \leq U,
\end{aligned}
$$

for some (given) constants $F>0, U>0$. Then, for any $\tau \in(0, \min \{1, T-S\})$, we have

$$
\left\|\vartheta_{t}\right\|_{L^{2}(S+\tau, T ; H)}+\alpha\left\|\vartheta_{t}\right\|_{L^{2}\left(S+\tau, T ; H_{\Gamma}\right)}+\|u\|_{L^{\infty}(S+\tau, T ; V)}+\beta\|\vartheta\|_{L^{\infty}\left(S+\tau, T ; V_{\Gamma}\right)} \leq Q\left(F, U, \tau^{-1}, T-S\right) .
$$

Proof. We just consider the case when $\alpha, \beta>0$, which is the most difficult one. For simplicity, we can then set $\alpha=\beta=1$ and write

$$
\int_{\bar{\Omega}} v:=\int_{\Omega} v+\int_{\Gamma} v
$$

whenever $v$ is, say, an element of $\mathcal{H}$. Then, we test both (3.2) and (3.3) by $u_{t}=\vartheta_{t} / \vartheta^{2}$ (note that we are always assuming the solution to be smooth enough for our purposes). Then, standard integrations by parts lead to

$$
\frac{1}{2} \frac{\mathrm{d}}{\mathrm{d} t}\left(\|\nabla u\|^{2}+\int_{\Gamma} \frac{\left|\nabla_{\Gamma} \vartheta\right|^{2}}{\vartheta^{2}}\right)+\int_{\bar{\Omega}} \frac{\vartheta_{t}^{2}}{\vartheta^{2}} \leq \int_{\Gamma} \frac{\vartheta_{t}\left|\nabla_{\Gamma} \vartheta\right|^{2}}{\vartheta^{3}}+\int_{\Omega} f \frac{\vartheta_{t}}{\vartheta^{2}}
$$

Of course, using (3.68), the latter term can be simply estimated as follows:

$$
\int_{\Omega} f \frac{\vartheta_{t}}{\vartheta^{2}} \leq \frac{1}{4} \int_{\Omega} \frac{\vartheta_{t}^{2}}{\vartheta^{2}}+Q(U)\|f\|^{2} .
$$

The first term on the right hand side of (3.71) is more delicate. Actually, owing to standard interpolation inequalities, we have

$$
\begin{aligned}
\int_{\Gamma} \frac{\vartheta_{t}\left|\nabla_{\Gamma} \vartheta\right|^{2}}{\vartheta^{3}} & \leq Q(U)\left\|\vartheta_{t}\right\|_{\Gamma}\left\|\nabla_{\Gamma} \vartheta\right\|_{4, \Gamma}^{2} \leq Q(U)\left\|\vartheta_{t}\right\|_{\Gamma}\|\vartheta\|_{H^{2}(\Gamma)}\|\vartheta\|_{\infty, \Gamma} \\
& \leq Q(U)\left(\left\|\vartheta_{t}\right\|_{\Gamma}^{2}+\left\|-\Delta_{\Gamma} \vartheta\right\|_{\Gamma}^{2}+\|\vartheta\|_{\Gamma}^{2}\right) \\
& \leq Q(U)\left(\left\|\vartheta_{t}-\Delta_{\Gamma} \vartheta\right\|_{\Gamma}^{2}\right)+Q(U)-Q(U) \frac{\mathrm{d}}{\mathrm{d} t}\left\|\nabla_{\Gamma} \vartheta\right\|_{\Gamma}^{2} .
\end{aligned}
$$


Then, the last term is moved to the left hand side and will give a positive contribution. We now estimate the first term on the right hand side, which requires some work. Actually, we first notice that, comparing terms in equation (3.3), using the trace theorem and elliptic regularity results (cf., e.g., [7. Thm. 2.5.13 and Thm. 2.7.4]), for arbitrarily small but otherwise fixed $\delta \in(0,1 / 2)$, we obtain

$$
\begin{aligned}
Q(U)\left(\left\|\vartheta_{t}-\Delta_{\Gamma} \vartheta\right\|_{\Gamma}^{2}\right)+Q(U)\left\|\partial_{\boldsymbol{n}} u\right\|_{\Gamma}^{2} & \leq Q(U)\left\|\partial_{\boldsymbol{n}} u\right\|_{\Gamma}^{2} \leq Q(U)\|u\|_{H^{\frac{3}{2}+\delta}(\Omega)}^{2} \\
& \leq Q(U)\left(\|u\|^{2}+\|\Delta u\|_{H^{-\frac{1}{2}+\delta}(\Omega)}^{2}+\|u\|_{H^{1+\delta}(\Gamma)}^{2}\right) \\
& \leq Q(U)\left(1+\|\Delta u\|^{p}\|u\|^{2-p}+\|u\|_{H^{1}(\Gamma)}^{2-2 \delta}\|u\|_{H^{2}(\Gamma)}^{2 \delta}\right),
\end{aligned}
$$

for a suitable $p \in(0,2)$ depending on the choice of $\delta$. Then, using (3.2) and Young's inequality, we obtain

$$
\begin{aligned}
Q(U)\|\Delta u\|^{p}\|u\|^{2-p} & \leq Q(U)\|\Delta u\|^{p} \leq Q(U)\left\|\vartheta_{t}-f\right\|^{p} \\
& \leq \sigma \int_{\Omega} \frac{\vartheta_{t}^{2}}{\vartheta^{2}}+Q_{\sigma}(U)\left(1+\|f\|^{2}\right) .
\end{aligned}
$$

Moreover, comparing once more terms in (3.3) and noting in particular that

$$
\Delta_{\Gamma} u=\operatorname{div}_{\Gamma}\left(u^{2} \nabla_{\Gamma} \vartheta\right)
$$

we arrive at

$$
\begin{aligned}
\left\|\Delta_{\Gamma} u\right\|_{\Gamma} & \leq Q(U)\left\|\Delta_{\Gamma} \vartheta\right\|_{\Gamma}+Q(U)\left\|\nabla_{\Gamma} \vartheta\right\|_{4, \Gamma}^{2} \\
& \leq Q(U)\left\|\Delta_{\Gamma} \vartheta\right\|+Q(U)+Q(U)\left\|\Delta_{\Gamma} \vartheta\right\|_{\Gamma}\|\vartheta\|_{\infty, \Gamma} \\
& \leq Q(U)\left\|\Delta_{\Gamma} \vartheta\right\|_{\Gamma}+Q(U) .
\end{aligned}
$$

Hence, the last term in (3.74) can be controlled this way:

$$
\begin{aligned}
Q(U)\|u\|_{H^{1}(\Gamma)}^{2-2 \delta}\|u\|_{H^{2}(\Gamma)}^{2 \delta} & \leq Q_{\sigma}(U)\|u\|_{H^{1}(\Gamma)}^{2}+\sigma\left\|\Delta_{\Gamma} u\right\|_{\Gamma}^{2} \\
& \leq Q_{\sigma}(U)\|u\|_{H^{1}(\Gamma)}^{2}+\sigma Q(U)\left\|\Delta_{\Gamma} \vartheta\right\|_{\Gamma}^{2}+\sigma Q(U) \\
& \leq Q_{\sigma}(U)\|u\|_{H^{1}(\Gamma)}^{2}+\sigma Q(U)\left\|\vartheta_{t}\right\|_{\Gamma}^{2}+\sigma Q(U)\left\|\partial_{\boldsymbol{n}} u\right\|_{\Gamma}^{2}+\sigma Q(U) \\
& \leq Q_{\sigma}(U)\|\vartheta\|_{H^{1}(\Gamma)}^{2}+\sigma Q(U) \int_{\Gamma} \frac{\vartheta_{t}^{2}}{\vartheta^{2}}+\sigma Q(U)\left\|\partial_{\boldsymbol{n}} u\right\|_{\Gamma}^{2}+\sigma Q(U),
\end{aligned}
$$

where $\sigma>0$ is small and $Q_{\sigma}$ also depends on $\sigma$. In particular, taking $\sigma$ small enough both in (3.75) and in (3.78), and collecting (3.72)-(3.78), (3.71) gives

$$
\begin{aligned}
\frac{1}{2} \frac{\mathrm{d}}{\mathrm{d} t} & \left(\|\nabla u\|^{2}+\int_{\Gamma} \frac{\left|\nabla_{\Gamma} \vartheta\right|^{2}}{\vartheta^{2}}+Q(U)\left\|\nabla_{\Gamma} \vartheta\right\|_{\Gamma}^{2}\right)+\frac{1}{2} \int_{\bar{\Omega}} \frac{\vartheta_{t}^{2}}{\vartheta^{2}} \\
& \leq Q(U)\left(\|f\|^{2}+\|\vartheta\|_{H^{1}(\Gamma)}^{2}+1\right) .
\end{aligned}
$$

Now, thanks to (3.66), for any $\tau$ as in the statement, we can choose $\mathfrak{t} \in(S, S+\tau)$ such that

$$
\|\nabla u(\mathfrak{t})\|^{2}+\left\|\nabla_{\Gamma} \vartheta(\mathfrak{t})\right\|_{\Gamma}^{2} \leq Q\left(U, \tau^{-1}\right) .
$$

The thesis then follows by integrating (3.79) over $(\mathfrak{t}, T)$.

As before, for regular "initial" data, the property holds starting from the initial time:

Corollary 3.9. Let $S \in \mathbb{R}, T>S, \alpha \geq 0, \beta \geq 0$. If $\beta>0$, then let $\alpha>0$. Let $(u, \vartheta)$ be a couple of sufficiently smooth functions solving in a suitable sense system (3.2)-(3.3) over the time interval $(S, T)$. Moreover, let (3.66)-(3.68) hold together with

$$
u(S) \in V, \quad \beta \vartheta(S) \in V_{\Gamma}, \quad\|u(S)\|_{V}+\beta\|\vartheta(S)\|_{V_{\Gamma}}=: Z,
$$

for some $Z>0$. Then,

$$
\left\|\vartheta_{t}\right\|_{L^{2}(S, T ; H)}+\alpha\left\|\vartheta_{t}\right\|_{L^{2}\left(S, T ; H_{\Gamma}\right)}+\|u\|_{L^{\infty}(S, T ; V)}+\beta\|\vartheta\|_{L^{\infty}\left(S, T ; V_{\Gamma}\right)} \leq Q(F, U, Z, T-S) .
$$




\section{Existence for smooth data}

Based on the previous lemmas, we will prove here that, for sufficiently regular initial data and source term, Problem $(\mathrm{P})$ has a global solution in a rather good regularity class.

\subsection{Local existence of smooth solutions}

We start by showing that, for regular data, a local in time smooth solution exists. For the sake of simplicity, we give the proof only in the case when both $\alpha$ and $\beta$ are strictly positive. Actually, the other cases can be treated with differences that are mainly of technical character.

Theorem 4.1. Let $\alpha>0, \beta>0$. Let $T>0$ and let

$$
\begin{aligned}
& f \in C^{0}([0, T] \times \bar{\Omega}), \\
& \vartheta_{0} \in H^{2}(\Omega), \quad \underline{\vartheta} \leq \vartheta_{0}(x) \leq \bar{\vartheta} \text { for all } x \in \bar{\Omega},
\end{aligned}
$$

for suitable constants $0<\underline{\vartheta}<\bar{\vartheta}$. Then, there exists $T_{0} \in(0, T]$ depending on $f$ and $\vartheta_{0}$ (and in particular on $\underline{\vartheta}, \bar{\vartheta})$, such that Problem $(\mathrm{P})$ admits a solution $(\vartheta, u)$ over the time interval $\left[0, T_{0}\right]$ satisfying

$$
\begin{aligned}
& \vartheta \in H^{1}\left(0, T_{0} ; \mathcal{H}\right) \cap C^{0}\left(\left[0, T_{0}\right] \times \bar{\Omega}\right), \quad \frac{\vartheta}{\overline{2}} \leq \vartheta(t, x) \leq 2 \bar{\vartheta} \text { for all }(t, x) \in\left[0, T_{0}\right] \times \bar{\Omega} \\
& u \in L^{2}\left(0, T_{0} ; H^{2}(\Omega)\right), \quad \vartheta \in L^{2}\left(0, T_{0} ; H^{2}(\Gamma)\right)
\end{aligned}
$$

Proof. We only sketch it, since it follows from more or less classical arguments for quasilinear parabolic problems. The key idea is to regularize the monotone function $\gamma(r)=-1 / r$ by introducing a function $\gamma_{R}: \mathbb{R} \rightarrow \mathbb{R}$ with the following properties

$$
\begin{array}{r}
\gamma_{R} \in C^{2}(\mathbb{R}), \quad \gamma_{R}^{\prime \prime} \in L^{\infty}(\mathbb{R}), \quad \gamma_{R}(r)=\gamma(r) \quad \forall r \in\left[\frac{\underline{\eta}}{2}, 2 \bar{\vartheta}\right], \\
\exists 0<m_{R}<M_{R} \quad \text { such that } m_{R} \leq \gamma_{R}^{\prime}(r) \leq M_{R} \quad \forall r \in \mathbb{R} .
\end{array}
$$

In other words, $\gamma_{R}$ coincides with $\gamma$ over an interval that is strictly larger than the range of the initial values of the problem. Outside that interval, we substitute it by a smooth and bi-Lipschitz approximation (e.g., we can take the first order Taylor expansion and then mollify). At this point, we can solve the problem by means of a fixed point argument. We just give the highlight of this procedure and set, for simplicity, $\alpha=\beta=1$.

As a first step, we take a function $\phi$ in the closed ball $\Phi$ of radius 1 of the space

$$
L^{2}\left(0, T_{0} ; H^{\varepsilon}(\Gamma)\right)
$$

where $\varepsilon \in(0,1 / 4)$ is fixed but otherwise arbitrary. Then, we consider the initial-value problem

$$
\eta_{t}-\Delta_{\Gamma} \eta=\phi,\left.\quad \eta\right|_{t=0}=\left.\vartheta_{0}\right|_{\Gamma}, \quad \text { on } \Gamma \text {. }
$$

and we claim that it admits a unique solution $\eta$ lying in a bounded closed ball of the space

$$
H^{1}\left(0, T_{0} ; H^{\varepsilon}(\Gamma)\right) \cap L^{2}\left(0, T_{0} ; H^{2+\varepsilon}(\Gamma)\right) .
$$

The simpler way to obtain this property is probably to prove it directly by interpolation. Actually, the regularity (4.7) of the datum $\phi$ implies the regularity (4.9) of the solution $\eta$ for $\varepsilon=0,1$ (this is classical and can be proved easily; notice that $\Gamma$ is a smooth manifold without boundary). Moreover, the solution operator $\phi \mapsto \eta$ is continuous for $\varepsilon=0,1$. Hence, by standard interpolation results we get continuity of the solution map for $\varepsilon \in(0,1 / 4)$. Notice also that, for such a choice of $\varepsilon$, the regularity of the boundary initial datum (which is known to lie in $H^{3 / 2}(\Gamma)$ in view of (4.2) and the trace theorem), is sufficient. Next, we observe that, again by interpolation, the space (4.9) is continuously embedded into $C^{0, a}\left(\left[0, T_{0}\right] \times \Gamma\right)$ for some $a>0$ depending on the choice of $\varepsilon$ (recall that $\Omega$ is smooth and its 
boundary $\Gamma$ is a 2 -dimensional manifold). Note also that this property may fail for $\varepsilon=0$. Thus, we can take $T_{0}$ so small that, additionally,

$$
\frac{3 \underline{\vartheta}}{4} \leq \eta(t, x) \leq \frac{3}{2} \bar{\vartheta} \text { for all }(t, x) \in\left[0, T_{0}\right] \times \Gamma .
$$

As a subsequent step, we solve the problem

$$
\begin{aligned}
& \vartheta_{t}-\Delta \gamma_{R}(\vartheta)=f,\left.\quad \vartheta\right|_{t=0}=\vartheta_{0}, \quad \text { in } \Omega, \\
& \gamma_{R}(\vartheta)=\gamma_{R}(\eta), \quad \text { on } \Gamma .
\end{aligned}
$$

This is a quasilinear parabolic system with bi-Lipschitz continuous nonlinearity $\gamma_{R}$ and Dirichlet boundary conditions. Then, by standard methods one may verify that it admits a unique solution $\vartheta$ such that

$$
\vartheta \in H^{1}\left(0, T_{0} ; H\right), \quad \gamma_{R}(\vartheta) \in L^{\infty}\left(0, T_{0} ; H^{1}(\Omega)\right) \cap L^{2}\left(0, T_{0} ; H^{2}(\Omega)\right) .
$$

For instance, the basic a priori estimate corresponding to the above regularity can be obtained testing the equation in (4.11) by the function $\partial_{t}\left(\gamma_{R}(\vartheta)-\mathcal{R}\left(\gamma_{R}(\eta)\right)\right)$, where $\mathcal{R}$ denotes the harmonic extension operator, namely

$$
-\Delta \mathcal{R}(v)=0 \text { in } \Omega, \quad \mathcal{R}(v)=v \text { on } \Gamma,
$$

where $v$ is, say, a function in $H_{\Gamma}$. Note that the regularity (4.9) of the trace and the $C^{2}$-regularity of $\gamma_{R}$ are essential for the sake of obtaining (4.13), as a direct check permits us to verify.

Then, being $\gamma_{R}$ bi-Lipschitz, $\partial_{t}\left(\gamma_{R}(\vartheta)\right)$ has the same $L^{2}$-regularity as $\vartheta_{t}$ (cf. (4.13)). Hence, we may conclude that $\gamma_{R}(\vartheta)$ lies in a bounded closed ball of the space

$$
H^{1}\left(0, T_{0} ; H\right) \cap L^{\infty}\left(0, T_{0} ; H^{1}(\Omega)\right) \cap L^{2}\left(0, T_{0} ; H^{2}(\Omega)\right) .
$$

Moreover, the equation in (4.11) is quasilinear and uniformly parabolic and it has uniformly bounded initial data (by (4.2) ), boundary data (by (4.10), and forcing term (by (4.1)). Thus, standard barrier arguments entail that, up to possibly taking a smaller initial time $T_{0}$ (in a way that only depends on the known norms of $\vartheta_{0}$ and $f$, on the regularized function $\gamma_{R}$, and on the truncation values $\underline{\vartheta}, \bar{\vartheta}$ ),

$$
\frac{\underline{\bar{V}}}{2} \leq \vartheta(t, x) \leq 2 \bar{\vartheta} \text { for all }(t, x) \in\left[0, T_{0}\right] \times \bar{\Omega}
$$

Next, let us notice that, by interpolation of Sobolev spaces (cf. once more [7, Chap. 2] for details), the space in (4.15) is continuously embedded into $H^{\delta}\left(0, T_{0} ; H^{2-2 \delta}(\Omega)\right)$ for any $\delta \in(0,1)$. Hence, at least for $\delta \in(0,1 / 4)$, we may apply the trace theorem [7, Thm. 2.7.4]. In particular, taking $\delta=1 / 8$, we obtain that the function $\partial_{\boldsymbol{n}} \gamma_{R}(\vartheta)$ lies in a bounded closed ball of the space

$$
H^{1 / 8}\left(0, T_{0} ; H^{1 / 4}(\Omega)\right),
$$

which, in view of the fact that $\varepsilon \in(0,1 / 4)$, is continuously and compactly embedded into the space in (4.7). Hence, the map

$$
\mathcal{T}: \Phi \rightarrow L^{2}\left(0, T_{0} ; H^{\varepsilon}(\Gamma)\right), \quad \mathcal{T}: \phi \mapsto \partial_{\boldsymbol{n}} \gamma(\vartheta)
$$

is compact. Next, we prove that, for $T_{0}$ small enough, $\mathcal{T}$ takes values into $\Phi$. Actually, interpolating the latter two spaces in (4.15), we obtain that $\gamma_{R}(\vartheta)$ lies in a bounded closed ball of $L^{16 / 7}\left(0, T_{0} ; H^{7 / 4}(\Omega)\right)$, whence, applying once more [7, Thm. 2.7.4], $\partial_{\boldsymbol{n}} \gamma_{R}(\vartheta)$ also lies in a bounded closed ball of the space $L^{16 / 7}\left(0, T_{0} ; H^{1 / 4}(\Gamma)\right)$. Moreover, the radius of that ball has the form $Q_{1}\left(\left\|\vartheta_{0}\right\|_{H^{2}(\Omega)}\right)$. In particular, the radius is independent of $T_{0}$ in view of the fact that all the constants appearing in the estimates performed so far are also independent of $T_{0}$ since they come from parabolic regularity theorems. In other words, we do not need to use Sobolev's embeddings (in which case the embedding constants may explode as the measure of the time interval $\left(0, T_{0}\right)$ becomes small). Hence, since $16 / 7>2$ and $\varepsilon \in(0,1 / 4)$, comparing with (4.7), we can take $T_{0}$ small enough so that $\mathcal{T}$ takes values into $\Phi$. Finally, 
by a number of standard checks one may verify that $\mathcal{T}$ is continuous. Hence, the Schauder fixed point theorem may be applied to $\mathcal{T}$. This provides a local solution to the system

$$
\begin{aligned}
& \vartheta_{t}-\Delta \gamma_{R}(\vartheta)=f, \quad \text { in } \Omega, \\
& \eta_{t}-\Delta_{\Gamma} \eta=-\partial_{\boldsymbol{n}} \gamma_{R}(\vartheta), \quad \text { on } \Gamma,
\end{aligned}
$$

plus the initial conditions and the boundary condition $\left.\vartheta\right|_{\Gamma}=\eta$. However, thanks to (4.16) and the latter (4.5), $\gamma_{R}(\vartheta)$ coincides with $\gamma(\vartheta)=-1 / \vartheta=: u$ everywhere in $\left[0, T_{0}\right] \times \bar{\Omega}$. Hence, $(\vartheta, u)$ is a local smooth solution to (3.2)-(3.3). This concludes the proof.

\subsection{Energy estimate}

In this section we prove the basic energy estimate satisfied by solutions of Problem (P). We start by recalling a generalized version of Poincaré's inequality (see for instance [18, Lemma 3.2] for a proof).

Lemma 4.2. Assume $\Omega$ is a bounded open subset of $\mathbb{R}^{d}$. Suppose $v \in W^{1,1}(\Omega)$ and $v \geq 0$ a.e. in $\Omega$. Then, setting $K:=\int_{\Omega}(\log v)^{+}$, the following estimate holds:

$$
\|v\|_{L^{1}(\Omega)} \leq|\Omega| e^{C_{1} K}+\frac{C_{2}}{|\Omega|}\|\nabla v\|_{L^{1}(\Omega)},
$$

the positive constants $C_{1}$ and $C_{2}$ depending only on $\Omega$.

Lemma 4.3 (Energy estimate). Let $\alpha \geq 0, \beta \geq 0$ and let (2.10)-(2.11) and (2.14) hold. Let $(\vartheta, u)$ be a sufficiently smooth solution to Problem (P). Then, we have

$$
\mathcal{E}(t)+\int_{0}^{t}\left(\|\nabla u\|^{2}+\beta\left\|\nabla_{\Gamma} \log \vartheta\right\|_{\Gamma}^{2}\right)+\|u\|_{L^{2}(t, t+1 ; V)}^{2} \leq Q\left(\mathbb{E}_{0},\|f\|_{L^{2}\left(0, t ; L^{6 / 5}(\Omega)\right)}\right), \quad \forall t \geq 0 .
$$

Proof. Test (2.6) and (2.7) with $1+u$. This gives

$$
\frac{\mathrm{d}}{\mathrm{d} t} \mathcal{E}(t)+\left(\|\nabla u\|^{2}+\beta\left\|\nabla_{\Gamma} \log \vartheta\right\|_{\Gamma}^{2}\right)=\int_{\Omega} f(1+u) .
$$

Then, using (2.14) and the Poincaré-Wirtinger inequality, we estimate the right hand side as follows:

$$
\int_{\Omega} f(1+u)=\int_{\Omega} f u=\int_{\Omega} f\left(u-m_{\Omega}(u)\right) \leq \frac{1}{2}\|\nabla u\|^{2}+c\|f\|_{L^{6 / 5}(\Omega)}^{2},
$$

with $c$ independent of time. Integrating (4.23) in time from 0 to $t$, we obtain

$$
\mathcal{E}(t)+\int_{0}^{t}\left(\frac{1}{2}\|\nabla u\|^{2}+\beta\left\|\nabla_{\Gamma} \log \vartheta\right\|_{\Gamma}^{2}\right) \leq \mathbb{E}_{0}+c\|f\|_{L^{2}\left(0, t ; L^{6 / 5}(\Omega)\right)}^{2}, \quad \forall t \geq 0 .
$$

To obtain the control on the full $V$-norm of $u$, we use Lemma 4.2. Thus, taking $v=1 / \vartheta=-u$ in (4.21), we infer

$$
\|u\|_{L^{1}(\Omega)} \leq|\Omega| e^{C_{1} \int_{\Omega} \log ^{-} \vartheta}+\frac{C_{2}}{|\Omega|}\|\nabla u\|_{L^{1}(\Omega)} .
$$

Hence, squaring, integrating over $(t, t+1)$, and using (4.25), we obtain

$$
\|u\|_{L^{2}\left(t, t+1 ; L^{1}(\Omega)\right)} \leq Q\left(\mathbb{E}_{0}\right) .
$$

The thesis follows combining (4.27) and (4.25). 


\subsection{Proof of global existence}

Our next aim is to prove that, for smooth initial data, the solution constructed in Section 4.1 has, in fact, a global in time character. We can treat all cases with the exception of $\alpha=0, \beta>0$. This case will be dealt with separately in Section 5.3 below.

Theorem 4.4. Let $\alpha \geq 0, \beta \geq 0$ and, if $\alpha=0$, then let $\beta=0$. Let (2.14) hold together with

$$
\vartheta_{0} \in \mathcal{V}, \quad \underline{\vartheta} \leq \vartheta_{0}(x) \leq \bar{\vartheta} \text { for all } x \in \bar{\Omega},
$$

for some $0<\underline{\vartheta}<\bar{\vartheta}$. Then, there exists a global solution to Problem $(\mathrm{P})$ satisfying, for all $T>0$,

$$
\begin{aligned}
& \vartheta \in H^{1}(0, T ; H) \cap L^{\infty}((0, T) \times \Omega) \cap L^{\infty}(0, T ; V), \\
& u \in H^{1}(0, T ; H) \cap L^{\infty}((0, T) \times \Omega) \cap L^{\infty}(0, T ; V) \cap L^{2}\left(0, T ; H^{3 / 2}(\Omega)\right), \\
& \alpha \eta \in H^{1}\left(0, T ; H_{\Gamma}\right), \quad \beta \eta \in L^{\infty}\left(0, T ; V_{\Gamma}\right) \cap L^{2}\left(0, T ; H^{2}(\Gamma)\right) .
\end{aligned}
$$

Moreover, if either $\alpha>0$ and $\beta>0$ or $\alpha=\beta=0$, then we have more precisely

$$
\vartheta \in L^{2}\left(0, T ; H^{2}(\Omega)\right), \quad u \in L^{2}\left(0, T ; H^{2}(\Omega)\right) .
$$

Proof. Let us fix (an arbitrary) $T>0$. In this proof we will allow the constants $c$ to depend on $T$. Indeed, we are working on a fixed interval and are not looking for uniform estimates at this level. In order to get a local solution via Theorem 4.1 we need to construct sequences of regularized initial and source data $\left\{\vartheta_{0, n}\right\}$ and $\left\{f_{n}\right\}$ satisfying, for all $n \in \mathbb{N}$, (4.1) and, respectively, (4.2). Moreover, we need $\vartheta_{0, n} \rightarrow \vartheta_{0}$ and $f_{n} \rightarrow f$ in proper ways. The details of the regularization procedure are sketched in Section 5.2 below, to which we refer the reader.

Then, thanks to Theorem 4.1 for all $n>0$ we have a solution to the $n$-Problem defined on some interval $\left(0, T_{0, n}\right)$, with $T_{0, n} \leq T$. We now deduce global in time estimates independent of $n$ and, for the sake of simplicity, we shall directly work on the time interval $(0, T)$ rather than on $\left(0, T_{0, n}\right)$. As usual, this can be justified a posteriori by means of standard extension arguments. To be more precise, one may first extend the approximate solution up to the final time $T$ by applying the uniform estimates at fixed $n$. Indeed, these estimates imply that, if $T_{0, n}^{\prime} \geq T_{0, n}$ is the maximal existence time of the approximate solution in the regularity class of Theorem 4.1, then the approximate solution cannot explode as $t \nearrow T_{0, n}^{\prime}$ (because the estimates are uniform) and consequently $T_{0, n}^{\prime}$ must coincide with the reference time $T$. Once the approximate solutions are proved to exist over $(0, T)$, then one may apply once more the estimates in order to let $n \nearrow \infty$ and remove the approximation.

That said, we can first apply Lemma 4.3, which gives the bound

$$
\left\|\vartheta_{n}\right\|_{L^{\infty}\left(0, T ; L^{1}(\Omega)\right)}+\alpha\left\|\eta_{n}\right\|_{L^{\infty}\left(0, T ; L^{1}(\Gamma)\right)}+\left\|u_{n}\right\|_{L^{2}(0, T ; V)}+\beta\left\|\log \eta_{n}\right\|_{L^{2}\left(0, T ; V_{\Gamma}\right)} \leq c,
$$

for $c>0$ independent of $n$. Indeed, from this point on, we go back to the notation $\eta_{n}$ when we indicate the boundary value of $\vartheta_{n}$. Next, we can apply Corollaries 3.2, 3.6 (with $S=0$ ), which give

$$
\left\|u_{n}\right\|_{L^{\infty}((0, T) \times \Omega)}+\left\|\vartheta_{n}\right\|_{L^{\infty}((0, T) \times \Omega)} \leq c .
$$

Clearly, the same uniform boundedness properties hold also for the traces on $\Gamma$.

Next, by Corollary 3.9 (here the restriction on $\alpha$ and $\beta$ comes into play), we have

$$
\left\|\left(\vartheta_{n}\right)_{t}\right\|_{L^{2}(0, T ; H)}+\alpha\left\|\left(\eta_{n}\right)_{t}\right\|_{L^{2}\left(0, T ; H_{\Gamma}\right)}+\left\|u_{n}\right\|_{L^{\infty}(0, T ; V)}+\beta\left\|\eta_{n}\right\|_{L^{\infty}\left(0, T ; V_{\Gamma}\right)} \leq c .
$$

Comparing terms in equation (3.2), we also obtain

$$
\left\|\Delta u_{n}\right\|_{L^{2}(0, T ; H)} \leq c .
$$

Moreover, in the case when $\beta>0$ (and hence $\alpha>0$ ), we observe that

$$
\left.u_{n}\right|_{\Gamma}=-\frac{1}{\eta_{n}} \text { and }\left\|\frac{1}{\eta_{n}}\right\|_{L^{2}\left(0, T ; V_{\Gamma}\right)} \leq c,
$$


the latter bound following from (4.33)-(4.34). Hence, by standard regularity results for elliptic problems with Dirichlet boundary conditions (see, e.g., [7, Theorem 3.1.5]), we obtain

$$
\left\|u_{n}\right\|_{L^{2}\left(0, T ; H^{3 / 2}(\Omega)\right)} \leq c .
$$

Thanks to (4.33) and (4.36), we can apply the trace theorem [7. Theorem 2.7.7], which yields

$$
\left\|\partial_{\boldsymbol{n}} u_{n}\right\|_{L^{2}\left(0, T ; H_{\Gamma}\right)} \leq c .
$$

Consequently, using the regularity of the boundary initial datum (4.28) we obtain

$$
\left\|\eta_{n, t}\right\|_{L^{2}\left(0, T ; H_{\Gamma}\right)}+\left\|\eta_{n}\right\|_{L^{2}\left(0, T ; H^{2}(\Gamma)\right)} \leq c .
$$

Next, we observe that

$$
\Delta_{\Gamma}\left(-\frac{1}{\eta_{n}}\right)=\frac{1}{\eta_{n}^{2}} \Delta_{\Gamma} \eta_{n}-2 \frac{\left|\nabla_{\Gamma} \eta_{n}\right|^{2}}{\eta_{n}^{3}} .
$$

Thus, using the boundary analogue of (4.34), (4.40), and the Gagliardo-Nirenberg inequality

$$
\|v\|_{W^{1,4}} \leq c\|v\|_{H^{2}}^{1 / 2}\|v\|_{L^{\infty}}^{1 / 2}+c\|v\|_{L^{\infty}},
$$

which gives that $\left|\nabla_{\Gamma} \eta_{n}\right|^{2} \in L^{2}(\Gamma)$ uniformly w.r.t. $n$, we readily obtain that

$$
\left\|\frac{1}{\eta_{n}}\right\|_{L^{2}\left(0, T ; H^{2}(\Gamma)\right)} \leq c
$$

whence (4.38) can be improved to

$$
\left\|u_{n}\right\|_{L^{2}\left(0, T ; H^{2}(\Omega)\right)} \leq c
$$

and the same bound holds for $\left\{\vartheta_{n}\right\}$ thanks again to (4.34) and (4.42).

Let us now deal with the case when $\beta=0$. Then, if it is also $\alpha=0$, we are just dealing with no-flux conditions. Hence, from (4.36) we directly deduce (4.44) and, consequently, (4.32). If, instead, $\beta=0$ and $\alpha>0$, we then have that $\partial_{\boldsymbol{n}} u_{n}=-\alpha \eta_{n, t}$, so that, from (4.35), (4.36), and regularity results for Neumann elliptic boundary value problems, we deduce once more (4.38). However, it does not seem possible to arrive at (4.44) since we do not have sufficient regularity of $\partial_{\boldsymbol{n}} u_{n}$.

In all cases, standard applications of the Aubin-Lions lemma permit to take the limit $n \nearrow \infty$ and obtain (up to the extraction of a subsequence) existence of a triplet $(\vartheta, \eta, u)$ solving Problem (P) and complying with the regularity properties (4.29)-4.31) and, possibly, 4.32). In particular, it is worth noting that, in the case $\alpha=\beta=0$ there is no boundary function $\eta$. Otherwise, $\eta$ coincides with the trace of $\vartheta$ thanks to the quoted regularity properties and to continuity of the trace operator. The proof is concluded.

\section{$5 \quad$ Weak solutions}

\subsection{Existence for finite-energy data}

Theorem 5.1. Let assumptions (2.10)-(2.11) and (2.14) hold and let us assume that, if $\alpha=0$, then $\beta=0$. Then, Problem (P) admits at least one energy solution $(\vartheta, u)$ satisfying the uniform energy estimate

$$
\mathcal{E}(t)+\int_{0}^{t}\left(\frac{1}{2}\|\nabla u\|^{2}+\beta\left\|\nabla_{\Gamma} \log \vartheta\right\|_{\Gamma}^{2}\right) \leq \mathbb{E}_{0}+c\|f\|_{L^{2}\left(0, t ; L^{6 / 5}(\Omega)\right)}^{2}, \quad \forall t \geq 0 .
$$

and the further regularity property

$$
\|u(t)\|_{L^{\infty}(\Omega)} \leq Q\left(\mathbb{E}_{0}, M_{\epsilon}, \tau^{-1}\right) \quad \text { for a.e. } t \geq \tau, \quad \tau \in(0,1),
$$

where we have set

$$
M_{\varepsilon}:=\|f\|_{L^{2}\left(0,+\infty ; L^{6 / 5}(\Omega)\right)}+\|f\|_{\mathcal{T}^{2}\left(0,+\infty ; L^{3+\epsilon}(\Omega)\right)} .
$$


Proof. As before, we start with the case when $\alpha$ and $\beta$ are both strictly positive. The other cases can be treated with small variants that will be outlined at the end of the proof. In order to apply Theorem 4.4, we consider a sequence of initial data $\left\{\vartheta_{0, n}\right\}$ such that, as $n \nearrow \infty$,

$$
\begin{aligned}
& \vartheta_{0, n} \in \mathcal{V}, \quad 0<\underline{\vartheta}_{n} \leq \vartheta_{0, n}(x) \leq \bar{\vartheta}_{n}<+\infty, \quad \text { a.e. in } \Omega \text { and on } \Gamma, \\
& \vartheta_{0, n} \rightarrow \vartheta_{0} \quad \text { strongly in } L^{1}(\Omega),\left.\quad \vartheta_{0, n}\right|_{\Gamma} \rightarrow \eta_{0} \quad \text { strongly in } L^{1}(\Gamma), \\
& \limsup _{n \nearrow \infty}\left(\int_{\Omega} \log ^{-} \vartheta_{0, n}+\int_{\Gamma} \log ^{-} \vartheta_{0, n}\right)<\infty .
\end{aligned}
$$

The construction of such a sequence is sketched in Section 5.2 below. Notice that we do not need to approximate $f$. Then, for any $n \geq 0$, the assumptions of Theorem 4.4 are fulfilled and we have existence of a "smooth" solution $\left(\vartheta_{n}, u_{n}\right)$. Moreover, thanks to the energy estimate in Lemma 4.3 (cf. in particular (4.22) ), the assumptions of Lemma 3.1 are satisfied over the generic time interval $(t, t+2)$. Hence, thanks to (3.6), we obtain

$$
\left\|u_{n}(t)\right\|_{L^{\infty}(\Omega)} \leq Q\left(\mathbb{E}_{0}, \tau^{-1}\right) \text { for a.e. } t \geq \tau>0
$$

where it is worth noting once more that here $Q$ does not depend on the final time $T$. Indeed, we are applying Lemma 3.1 on a time interval of fixed length 2. Hence, we obtain a bound which is uniform for large $T$.

At this point, we use $L^{1}$-techniques in order to take the limit $n \nearrow \infty$. To this aim, we work on the generic interval $(0, T)$ and rewrite the approximate equation (2.6) in the equivalent form (which is possible since $u_{n}$ has the good regularity properties (4.30)

$$
\partial_{t} u_{n}=u_{n}^{2} \Delta u_{n}+u_{n}^{2} f
$$

Then, we test (5.8) with $\varphi \in L^{\infty}\left(0, T ; H_{0}^{s}(\Omega)\right)$, where we choose $s>\frac{3}{2}$ so that $H_{0}^{s}(\Omega) \subset C^{0}(\bar{\Omega})$, continuously. Note that, since $\varphi=0$ on $\Gamma$, we do not have to take into account the contribution of the boundary. Using (4.22) and (5.7), it is a standard matter to get

$$
\left\|u_{n, t}\right\|_{L^{1}\left(\tau, T ; H^{-s}\right)} \leq c .
$$

Next, for any $\varepsilon>0$ and $r \in \mathbb{R}$ we introduce the approximate $\operatorname{sign}$ function as $\operatorname{sign}_{\varepsilon}(v):=\frac{v}{\varepsilon+|v|}$. Then, we write the approximate equations (2.6) and (2.7) for the indexes $m$ and $n$, take the differences and test them, respectively, by $\operatorname{sign}_{\varepsilon}\left(u_{m}-u_{n}\right)$ and by $\operatorname{sign}_{\varepsilon}\left(u_{m, \Gamma}-u_{n, \Gamma}\right)$. Indeed, for any $m, n \in \mathbb{N}$ and $\varepsilon>0$, we have

$$
\operatorname{sign}_{\varepsilon}\left(u_{m}-u_{n}\right) \in L^{2}\left(0, T ; V_{\Gamma}\right)
$$

since $\operatorname{sign}_{\varepsilon}$ is Lipschitz. Then, we arrive at

$$
\begin{aligned}
& \left(\left(\vartheta_{m, t}-\vartheta_{n, t}\right), \operatorname{sign}_{\varepsilon}\left(u_{m}-u_{n}\right)\right)+\alpha\left(\left(\eta_{m, t}-\eta_{n, t}\right), \operatorname{sign}_{\varepsilon}\left(u_{m}-u_{n}\right)\right)_{\Gamma} \\
& \quad-\beta\left(\Delta_{\Gamma}\left(\eta_{m}-\eta_{n}\right), \operatorname{sign}_{\varepsilon}\left(u_{m}-u_{n}\right)\right)_{\Gamma} \leq 0 .
\end{aligned}
$$

Here, we used integration by parts to prove that

$$
-\left(\Delta\left(u_{m}-u_{n}\right), \operatorname{sign}_{\varepsilon}\left(u_{m}-u_{n}\right)\right) \geq-\left(\partial_{\boldsymbol{n}}\left(u_{m}-u_{n}\right), \operatorname{sign}_{\varepsilon}\left(u_{m}-u_{n}\right)\right)_{\Gamma},
$$

which is possible thanks to the good regularity of approximate solutions, to monotonicity of $\operatorname{sign}_{\varepsilon}$, and to (5.10). Thus, integrating (5.10) over $(0, t)$, for a generic $t \in(0, T]$, we obtain

$$
\begin{aligned}
& \int_{0}^{t}\left(\left(\vartheta_{m, t}-\vartheta_{n, t}\right), \operatorname{sign}_{\varepsilon}\left(u_{m}-u_{n}\right)\right)+\alpha \int_{0}^{t}\left(\left(\eta_{m, t}-\eta_{n, t}\right), \operatorname{sign}_{\varepsilon}\left(u_{m}-u_{n}\right)\right)_{\Gamma} \\
& \quad-\beta \int_{0}^{t}\left(\Delta_{\Gamma}\left(\eta_{m}-\eta_{n}\right), \operatorname{sign}_{\varepsilon}\left(u_{m}-u_{n}\right)\right)_{\Gamma} \leq 0 .
\end{aligned}
$$


Next, we take the limit $\varepsilon \searrow 0$ and notice that, since $v \mapsto-1 / v$ is monotone increasing, it turns out that $\operatorname{sign}_{0}\left(\vartheta_{m}-\vartheta_{n}\right)=\operatorname{sign}_{0}\left(u_{m}-u_{n}\right)$ almost everywhere in $(0, T) \times \Omega$ and $\operatorname{sign}_{0}\left(\eta_{m}-\eta_{n}\right)=\operatorname{sign}_{0}\left(u_{m}-u_{n}\right)$ almost everywhere in $(0, T) \times \Gamma$, where $\operatorname{sign}_{0}$ denotes the sign-like function with $\operatorname{sign}_{0}(0)=0$. Then,

$$
\begin{aligned}
\int_{0}^{t} & \left(\left(\vartheta_{m, t}-\vartheta_{n, t}\right), \operatorname{sign}_{0}\left(\vartheta_{m}-\vartheta_{n}\right)\right)+\alpha \int_{0}^{t}\left(\left(\eta_{m, t}-\eta_{n, t}\right), \operatorname{sign}_{0}\left(\eta_{m}-\eta_{n}\right)\right)_{\Gamma} \\
& -\beta \int_{0}^{t}\left(\Delta_{\Gamma}\left(\eta_{m}-\eta_{n}\right), \operatorname{sign}_{0}\left(\eta_{m}-\eta_{n}\right)\right)_{\Gamma} \leq 0 .
\end{aligned}
$$

Applying the Brezis-Strauss lemma (see 6, Lemma 2]), we can integrate by parts the boundary Laplacian, which gives a nonnegative contribution. Thus, we arrive at

$$
\left\|\vartheta_{m}(t)-\vartheta_{n}(t)\right\|_{1}+\alpha\left\|\eta_{m}(t)-\eta_{n}(t)\right\|_{1, \Gamma} \leq\left\|\vartheta_{0, m}-\vartheta_{0, n}\right\|_{1}+\alpha\left\|\vartheta_{0, m}-\vartheta_{0, n}\right\|_{1, \Gamma} .
$$

Taking the supremum for $t \in[0, T]$, we finally obtain

$$
\vartheta_{n} \rightarrow \vartheta \quad \text { strongly in } C^{0}\left([0, T] ; L^{1}(\Omega)\right), \quad \alpha \eta_{n} \rightarrow \alpha \eta \quad \text { strongly in } C^{0}\left([0, T] ; L^{1}(\Gamma)\right),
$$

for suitable limit functions $\vartheta$ and $\eta$. Moreover, using (4.22), (5.9), and the Aubin-Lions lemma, we obtain that, up to the extraction of a subsequence,

$$
u_{n} \rightarrow u \quad \text { weakly in } L^{2}(0, T ; V) \quad \text { and strongly in } L^{2}\left(\tau, T ; H^{1-\delta}(\Omega)\right),
$$

for all $\tau \in(0,1), \delta \in(0,1)$, and for some limit function $u$. In particular, taking a test function $\xi$ satisfying (2.20), we can test both (2.6) and (2.7) (at the $n$-level) by $\xi$ and integrate by parts. This gives the $n$-analogue of (2.21). Moreover, the convergence properties proved above permit to take the limit $n \nearrow \infty$ to show that (2.21) holds also at the limit level. Hence, to conclude the proof it remains to identify the functions $\vartheta$ and $\eta$ in terms of $u$. To do this, we first observe that, combining the first (5.16) with (5.17), it follows that $\vartheta=-1 / u$ almost everywhere in $(0, T) \times \Omega$.

Next, by (5.17) and continuity of the trace operator, we have, for all $\tau \in(0, T)$,

$$
\left(u_{n}\right)_{\Gamma} \rightarrow u_{\Gamma} \quad \text { strongly in } L^{2}\left(\tau, T ; H^{1 / 2-\delta}(\Gamma)\right),
$$

with $u_{\Gamma}$ denoting the trace of $u$. In particular, thanks to arbitrariness of $\tau$, pointwise convergence holds on $(0, T) \times \Gamma$, up to the extraction of a further subsequence. Being $\eta_{n}=-1 /\left(u_{n}\right)_{\Gamma}$ almost everywhere in $(0, T) \times \Gamma$ and for all $n \in \mathbb{N}$, we then deduce that

$$
\eta_{n} \rightarrow-\frac{1}{u_{\Gamma}} \quad \text { a.e. on }(0, T) \times \Gamma \text {. }
$$

Combining this with the second (5.16), we then obtain relation (2.19). This concludes the proof of the Theorem in the case $\alpha>0, \beta>0$.

Let us now consider the case $\alpha>0$ and $\beta=0$ (the case $\alpha=\beta=0$ is essentially already known and, in any case, it is simpler to treat). Since Lemma 3.1 does not depend on whether $\beta$ is zero or not, estimate (5.7) (and consequently (5.9)) still holds when $\beta=0$. Moreover, it is easy to check that the Cauchy argument (5.10)-(5.16) can be reproduced also when $\beta=0$. The proof of the Theorem is concluded.

Remark 5.2. Note that, in the case when $\alpha>0$ and $\beta>0, \eta_{0}$ need not be the trace of $\vartheta_{0}$. Thus, there is a boundary layer in the sense specified by (5.18)-(5.19). However, (2.19) still makes sense thanks to instantaneous regularization properties.

\subsection{Approximation of data}

In this part, we sketch the approximation of data needed in the proofs of Theorems 4.4 and 5.1 Since the procedures are rather standard, we just give the highlights without entering too much into details.

Approximation of data for Theorem 4.4. We start with the initial data. Let us given $\vartheta_{0} \in \mathcal{V}$ such that $\underline{\vartheta} \leq \vartheta_{0}(x) \leq \bar{\vartheta}$ for all $x \in \bar{\Omega}$ (as in the statement, see (4.28)), and let $\eta_{0}$ be its trace on $\Gamma$. Since we want to apply Theorem 4.1 (cf., in particular, (4.2)), for any $n \in \mathbb{N}$ we need to have an approximate datum $\vartheta_{0, n}$ (with trace $\eta_{0, n}$ ) such that $\vartheta_{0, n} \in H^{2}(\Omega)$ for all $n$ and 
1. $\vartheta_{0, n} \stackrel{n \nearrow+\infty}{\longrightarrow} \vartheta_{0}$ in $\mathcal{V}$

2. $\underline{\vartheta} \leq \vartheta_{0, n}(x) \leq \bar{\vartheta}$ for all $x \in \bar{\Omega}$.

To construct $\vartheta_{0, n}$ we then consider the elliptic problem

$$
\left\{\begin{array}{l}
\vartheta_{0, n}-\frac{1}{n} \Delta \vartheta_{0, n}=\vartheta_{0} \text { in } \Omega, \\
-\frac{1}{n} \partial_{n} \vartheta_{0, n}=-\frac{1}{n} \Delta_{\Gamma} \eta_{0, n}+\eta_{0, n}-\eta_{0} \quad \text { on } \Gamma,\left.\quad \vartheta_{0, n}\right|_{\Gamma}=\eta_{0, n} .
\end{array}\right.
$$

Then, the standard elliptic theory gives that, for any fixed $n, \vartheta_{0, n}$ lies in $H^{2}(\Omega)$ and $\eta_{0, n}$ lies in $H^{2}(\Gamma)$.

To prove the convergence $\vartheta_{0, n} \rightarrow \vartheta_{0}$, we test (5.20) $\left.\right|_{\Omega}$ by $\left(\vartheta_{0, n}-\Delta \vartheta_{0, n}\right)$ and, correspondingly, $\left.(5.20)\right|_{\Gamma}$ by $\left(1+n^{-1}\right) \eta_{0, n}-\eta_{0}$. Then, by integrations by parts and Young's inequality, it is not difficult to obtain

$$
\left\|\vartheta_{0, n}\right\|_{\mathcal{V}}^{2}+2 n\left\|\eta_{0, n}-\eta_{0}\right\|_{\Gamma}^{2} \leq\left\|\vartheta_{0}\right\|_{\mathcal{V}}^{2}
$$

which clearly implies the desired convergence. On the other hand, to prove uniform boundedness of $\vartheta_{0, n}$, we use a maximum principle argument. We prove only the upper bound, the lower one being completely equivalent. For a generic $M>\bar{\vartheta}$, we test $\left.(\mathbf{5 . 2 0})\right|_{\Omega}$ by $\left(\vartheta_{0, n}-M\right)^{+}$. Integrating by parts the Laplacian and using (5.20) $\left.\right|_{\Gamma}$, we then obtain

$$
\int_{\Omega} \vartheta_{0, n}\left(\vartheta_{0, n}-M\right)^{+}+\int_{\Gamma} \eta_{0, n}\left(\eta_{0, n}-M\right)^{+} \leq \int_{\Omega} \vartheta_{0}\left(\vartheta_{0, n}-M\right)^{+}+\int_{\Gamma} \eta_{0}\left(\eta_{0, n}-M\right)^{+} .
$$

Adding to both sides the quantity $-M \int_{\Omega}\left(\vartheta_{0, n}-M\right)^{+}-M \int_{\Gamma}\left(\eta_{0, n}-M\right)^{+}$, we arrive at

$$
\left\|\left(\vartheta_{0, n}-M\right)^{+}\right\|^{2}+\left\|\left(\eta_{0, n}-M\right)^{+}\right\|_{\Gamma}^{2} \leq \int_{\Omega}\left(\vartheta_{0}-M\right)\left(\vartheta_{0, n}-M\right)^{+}+\int_{\Gamma}\left(\eta_{0}-M\right)\left(\eta_{0, n}-M\right)^{+} \leq 0 .
$$

This clearly gives the desired inequality $\vartheta_{0, n}(x) \leq \bar{\vartheta}$ for all $x \in \bar{\Omega}$.

Concerning the source term, recalling that $f$ satisfies (2.14), a combination of truncation and mollification techniques, together with a suitable correction of the spatial mean values permits to construct a family of functions $\left\{f_{n}\right\}, n \in \mathbb{N}$, such that

$$
\begin{aligned}
& f_{n} \in C^{0}([0, T] \times \bar{\Omega}), \quad\left\|f_{n}\right\|_{L^{\infty}((0, T) \times \Omega)} \leq n, \\
& f_{n} \rightarrow f \quad \text { strongly in } L^{1}((0, T) \times \Omega), \\
& \left(f_{n}(t)\right)_{\Omega}=0 \text { for all } t \in[0, T] \\
& \left\|f_{n}\right\|_{L^{2}\left(\left(0, T ; L^{3+\epsilon}(\Omega)\right)\right.} \leq c
\end{aligned}
$$

The details of this construction are left to the reader. Notice that (5.22)-(5.25) suffice both to apply Theorem 4.1 at the level $n$ to get a local smooth solution and to perform the estimates of Theorem 4.4 uniformly in $n$ in order to let $n \rightarrow \infty$.

Approximation of data for Theorem 5.1. We detail such an approximation just in the case $\alpha>0$, which is a little bit trickier. So, let $\left(\vartheta_{0}, \eta_{0}\right) \in L^{1}(\Omega) \times L^{1}(\Gamma)$ such that $\log \vartheta_{0} \in L^{1}(\Omega)$ and $\log \eta_{0} \in L^{1}(\Gamma)$. We need to construct $\vartheta_{0, n}$ in such a way that properties (5.4)-(5.5) hold. Then, we consider first the function $\vartheta_{0}$. Using that $\Gamma$ is smooth, we can first extend it (e.g., by reflection) to a neighbourhood $\Omega_{*}$ of $\bar{\Omega}$. It is then clear that the new function, note it as $\vartheta_{0, *}$, lies in $L^{1}\left(\Omega_{*}\right)$; moreover, $\log \vartheta_{0, *} \in L^{1}\left(\Omega_{*}\right)$. Next, we truncate $\vartheta_{0, *}$, setting

$$
\vartheta_{0, n}^{(1)}:=\min \left\{\max \left\{\vartheta_{0, *}, \frac{1}{n}\right\}, n\right\}, \quad \text { a.e. in } \Omega_{*} .
$$

Finally, we regularize, setting $\vartheta_{0, n}^{(2)}:=\rho_{n} * \vartheta_{0, n}^{(1)}$, where $\left\{\rho_{n}\right\}$ is a suitable sequence of smooth and compactly supported mollifiers. Then, straightforward checks (based on the properties of convolutions and on Lebesgue's theorem) and a standard diagonal argument permit to verify that $\vartheta_{0, n}^{(2)}$ is smooth 
and tends to $\vartheta_{0}$ strongly in $L^{1}(\Omega)$. Moreover, based on Jensen's inequality, it is not difficult to verify that, for some $c>0$ independent of $n$,

$$
\int_{\Omega} \log ^{-} \vartheta_{0, n}^{(2)} \leq c\left(1+\int_{\Omega} \log ^{-} \vartheta_{0}\right)
$$

Finally, we pass to the boundary component. First of all, thanks to smoothness of $\Gamma$, we can find $\epsilon>0$ such that $\eta_{0}$ can be extended (e.g., constantly along directions orthogonal to $\Gamma$ ) to a function $\eta_{0, *}$ defined on a neighbourhood $\Gamma_{\delta}:=\left\{x \in \mathbb{R}^{3}: d(x, \Gamma) \leq \delta\right\}$ of $\Gamma$. Thanks to Fubini's theorem, it is then clear that both $\eta_{0, *}$ and $\log \eta_{0, *}$ lie in $L^{1}\left(\Gamma_{\delta}\right)$. Then, we truncate $\eta_{0, *}$ (as in (5.26) ) obtaining $\eta_{0, n}^{(1)}$ (which can be seen as a function defined on the whole of $\mathbb{R}^{3}$ ). Next, we mollify $\eta_{0, n}^{(1)}$, introducing $\eta_{0, n}^{(2)}:=\rho_{n} * \eta_{0, n}^{(1)}$, for $\rho_{n}$ supported, say, on the ball $\bar{B}(0,1 / n)$. Finally, we take a cutoff function $\psi_{n} \in C^{\infty}\left(\mathbb{R}^{3} ;[0,1]\right)$ such that $\psi_{n}$ is identically one on $\Gamma_{1 / 2 n}$ and $\psi_{n}$ is supported on $\Gamma_{1 / n}$. Then, we set $\eta_{0, n}^{(3)}:=\eta_{0, n}^{(2)} \psi_{n}$ in such a way that $\eta_{0, n}^{(3)}$ belongs to $C^{\infty}\left(\mathbb{R}^{3}\right)$ and $\eta_{0, n}^{(3)}$ tends to 0 in $L^{1}(\Omega)$, while its trace tends to $\eta_{0}$ in $L^{1}(\Gamma)$. Moreover, as above, one can check that $\log ^{-}\left(\eta_{0, n}^{(3)}\right)$ is uniformly controlled in $L^{1}(\Gamma)$ in the sense of (5.27). Then, the required approximation of $\vartheta_{0}$ is obtained simply taking $\vartheta_{0, n}:=\left(1-\psi_{n}\right) \vartheta_{0, n}^{(2)}+\eta_{0, n}^{(3)}$.

\subsection{Solutions with regularizing effects for $\vartheta$}

In this last part, we extend the previous results in three directions. First, we prove that, if $\vartheta_{0}$ (and, possibly, $\eta_{0}$ ) enjoy higher summability properties, then there exist weak solutions whose component $\vartheta$ satisfies time-regularization properties in the spirit of Lemmas 3.4 and 3.5. Second, we demonstrate that, under the same type of conditions on the initial data, existence holds also for $\alpha=0$ and $\beta>0$ (recall that we could not deal with this case for $L^{1}$ initial data, cf. Theorem 5.11). Third, we see that uniqueness holds in the class of solutions with regularizing effects. We start with analyzing regularity:

Theorem 5.3. Let assumptions (2.10)-(2.11) and (2.14) hold and let in addition, for some $\epsilon \in(0,1)$,

$$
f \in L^{2}\left(0,+\infty ; L^{3+\epsilon}(\Omega)\right), \quad N_{\varepsilon}:=\|f\|_{L^{2}\left(0,+\infty ; L^{3+\epsilon}(\Omega)\right)} .
$$

Moreover, if $\alpha>0$ and $\beta=0$, let

$$
\vartheta_{0} \in L^{4+\epsilon}(\Omega), \quad \alpha \vartheta_{0} \in L^{4+\epsilon}(\Gamma)
$$

whereas in the other cases let

$$
\vartheta_{0} \in L^{3+\epsilon}(\Omega), \quad \alpha \vartheta_{0} \in L^{3+\epsilon}(\Gamma)
$$

Then, Problem (P) admits at least one energy solution $(\vartheta, u)$ satisfying (5.1), (5.2), together with the regularization estimate

$$
\|\vartheta(t)\|_{L^{\infty}(\Omega)} \leq Q\left(\mathbb{E}_{0+\epsilon}, N_{\varepsilon}, \tau^{-1}\right) \quad \forall t \geq \tau, \quad \tau \in(0,1)
$$

where we have set

$$
\mathbb{E}_{0+\epsilon}:=\mathcal{E}\left(\vartheta_{0}\right)+\left\|\vartheta_{0}\right\|_{L^{3+\epsilon}(\Omega)}^{3+\epsilon}+\alpha\left\|\vartheta_{0}\right\|_{L^{3+\epsilon}(\Gamma)}^{3+\epsilon},
$$

the exponents $3+\epsilon$ being all replaced by $4+\epsilon$ in the case when $\alpha>0$ and $\beta=0$. Moreover, in all cases with the exception of $\alpha=0$ and $\beta>0$, we have

$$
\begin{gathered}
\left\|\vartheta_{t}\right\|_{L^{2}(t, t+1 ; H)}+\alpha\left\|\eta_{t}\right\|_{L^{2}\left(t, t+1 ; H_{\Gamma}\right)}+\|u\|_{L^{\infty}(t,+\infty ; V)}+\beta\|\eta\|_{L^{\infty}\left(t, \infty ; V_{\Gamma}\right)} \\
\leq Q\left(\mathbb{E}_{0+\epsilon}, N_{\epsilon}, \tau^{-1}\right) \quad \forall t \geq \tau, \quad \tau \in(0,1) .
\end{gathered}
$$

Moreover, estimates (5.31) and (5.33) are uniform for large values of $t$. In other words, the expression of $Q$ is independent of $t$. 
Proof. For the sake of simplicity, we just prove the theorem by directly working on the "limit" solutions without referring to an explicit approximation scheme. That said, we first observe that the energy estimate (4.22) still holds. Moreover, we can still rely on the conclusion of Lemma 3.1. Next, thanks to assumption (5.28), we can apply Lemma 3.3 over the generic time interval $(0, T)$. Note that the estimates provided by Lemma 3.3 are uniform with respect to $T$. We then obtain

$$
\|\vartheta(t)\|_{L^{3+\epsilon}(\Omega)}+\alpha\|\vartheta(t)\|_{L^{3+\epsilon}(\Gamma)} \leq Q\left(N_{\varepsilon}, \mathbb{E}_{0+\epsilon}\right)
$$

(here and below, $3+\epsilon$ is replaced by $4+\epsilon$ in case $\alpha>0$ and $\beta=0$ ). Hence, for $t \geq \tau>0$, we can apply Lemma 3.4 (or Lemma 3.5) over the generic time interval $(t, t+2)$, which has fixed finite length. This gives (5.31).

Finally, in all cases with the exception of $\alpha=0$ and $\beta>0$, we can apply Lemma 3.8 over the generic time interval $(t, t+1)$ where $t \geq \tau>0$, which gives (5.33).

Then, to conclude the proof, it just remains to show that, in the case when $\alpha=0$ and $\beta>0$ (that we set equal to 1 for simplicity), a weak solution still exists under the above assumptions. To this aim, we consider the system

$$
\begin{aligned}
& \vartheta_{n, t}-\Delta u_{n}=f, \quad \vartheta_{n}=-1 / u_{n}, \quad \text { in } \Omega, \\
& \frac{1}{n} \eta_{n, t}-\Delta_{\Gamma} \eta_{n}=-\partial_{n} u, \quad \text { on } \Gamma,
\end{aligned}
$$

complemented with the usual initial conditions. Then, for all $n \in \mathbb{N}$, there exists at least one weak solution $\left(\vartheta_{n}, u_{n}\right)$. Moreover, thanks to (5.33) and to regularity arguments similar to those performed in Section 4.3. $\left(\vartheta_{n}, u_{n}\right)$ is smooth enough in order for the system to make sense in the above "strong" form, at least on time intervals of the form $(\tau, T)$ for all $\tau>0$.

In addition to that, we still have estimates (5.1) and (5.2). Moreover, it is worth noting that (5.2) holds independently of $n$. Indeed, looking back at the proof of Lemma 3.1 it is immediate to check that estimate (3.6) is independent of $\alpha$. Actually, when one performs the iteration argument (see (3.29) ) one simply has a functional $J_{i}$ that depends on $\alpha$, but the bound for such a functional remains unchanged. The same holds when applying Lemma 3.4. In conclusion, we have the uniform bound

$$
\left\|\vartheta_{n}(t)\right\|_{L^{\infty}(\Omega)}+\left\|u_{n}(t)\right\|_{L^{\infty}(\Omega)} \leq Q\left(\mathbb{E}_{0+\epsilon}, N_{\varepsilon}, \tau^{-1}\right) \quad \forall t \geq \tau, \quad \tau \in(0,1) .
$$

Thus, thanks also to Lemma 3.3, for any $\tau \in(0,1), T \geq \tau$, we have

$$
\vartheta_{n} \rightarrow \vartheta \quad \text { weakly star in } L^{\infty}\left(0, T ; L^{3+\epsilon}(\Omega)\right) \text { and weakly star in } L^{\infty}((\tau, T) \times \Omega) .
$$

Moreover, since $\beta>0$, as an additional consequence of estimate (3.39) (with $p=3+\epsilon$ ) we have

$$
\left\|\vartheta_{n^{\frac{1+\epsilon}{2}}}\right\|_{L^{2}(0, T ; V)}+\left\|\eta_{n^{\frac{3+\epsilon}{2}}}\right\|_{L^{2}\left(0, T ; V_{\Gamma}\right)} \leq c .
$$

In particular, being

$$
\nabla \vartheta_{n}=\frac{2}{1+\epsilon} \vartheta_{n^{\frac{1-\epsilon}{2}}}^{\nabla} \vartheta_{n^{\frac{1+\epsilon}{2}}}
$$

we have, from (5.38)-(5.39),

$$
\left\|\nabla \vartheta_{n}\right\|_{L^{2}\left(0, T ; L^{\frac{3+\epsilon}{2}}(\Omega)\right)} \leq c\left\|\nabla \vartheta_{n^{\frac{1+\epsilon}{2}}}\right\|_{L^{2}(0, T ; H)}\left\|\vartheta_{n^{\frac{1-\epsilon}{2}}}\right\|_{L^{\infty}\left(0, T ; L^{\frac{6+2 \epsilon}{1-\epsilon}}(\Omega)\right)} \leq c .
$$

In particular, this fact tells us that, in the present regularity setting, $\eta_{n}$ can be directly seen as the trace of $\vartheta_{n}$. More precisely, applying the trace theorem, we have

$$
\left\|\eta_{n}\right\|_{L^{2}\left(0, T ; W^{\frac{1+\epsilon}{3+\epsilon}}, \frac{3+\epsilon}{2}(\Gamma)\right)} \leq c
$$

On the other hand, testing (3.2) by a generic function $\phi \in H_{0}^{1}(\Omega)$ of unit norm and recalling (5.1) and (5.28), we obtain

$$
\vartheta_{n, t} \rightarrow \vartheta_{t} \quad \text { weakly in } L^{2}\left(0, T ; H^{-1}(\Omega)\right) \text {. }
$$


Thus, using (5.41), (5.43) and the Aubin-Lions lemma, we infer

$$
\vartheta_{n} \rightarrow \vartheta \quad \text { strongly in } L^{2}\left(0, T ; W^{1-\sigma, \frac{3+\epsilon}{2}}(\Omega)\right)
$$

for all $\sigma \in(0,1)$. Hence, by continuity of the trace operator,

$$
\eta_{n} \rightarrow \eta=\left.\vartheta\right|_{\Gamma} \quad \text { strongly in } L^{2}\left(0, T ; L^{p}(\Gamma)\right) \text { for some } p>1 .
$$

Thus, writing the weak formulation (2.21) of system (5.35)-(15.36), it is immediate to check that one can take the limit $n \rightarrow \infty$ therein. In particular, it can be standardly proved that

$$
u_{n} \rightarrow u=-1 / \vartheta \quad \text { strongly in } L^{p}\left(0, T ; L^{p}(\Omega)\right) \text {, say, for all } p \in[1,2) .
$$

Hence, in place of (2.19), we can now directly write $\eta=\left.\vartheta\right|_{\Gamma}$ in the sense of traces and a.e. in $(0, T)$. We may also notice that, thanks to the additional regularity properties coming from (5.41)-(5.42), we could even relax a bit the requirements (2.20) on the test function $\xi$ (we omit the details). The proof is concluded.

Finally, let us come to uniqueness:

Theorem 5.4. Let (2.10)-(2.11) and (2.14) hold. Let $\left(\vartheta_{1}, u_{1}\right)$ and $\left(\vartheta_{2}, u_{2}\right)$ be a couple of weak solutions to Problem (P) over some interval $(0, T)$ in the sense of Definition 2.1, both satisfying (5.31) and (5.33) and emanating from the same initial datum $\vartheta_{0}$. Then $\left(\vartheta_{1}, u_{1}\right)$ and $\left(\vartheta_{2}, u_{2}\right)$ coincide over $(0, T)$.

Proof. In view of the fact that $\left(\vartheta_{1}, u_{1}\right)$ and $\left(\vartheta_{2}, u_{2}\right)$ are smooth for strictly positive times, we can proceed as in Section 5.1 testing the difference of the equations by $\operatorname{sign}_{\varepsilon}\left(u_{1}-u_{2}\right)$. We then integrate over $(\tau, T)$ for $\tau>0$ and arrive at the analogue of (5.13), namely

$$
\begin{aligned}
& \int_{\tau}^{t}\left(\left(\vartheta_{1, t}-\vartheta_{2, t}\right), \operatorname{sign}_{\varepsilon}\left(u_{1}-u_{2}\right)\right)+\alpha \int_{\tau}^{t}\left(\left(\eta_{1, t}-\eta_{2, t}\right), \operatorname{sign}_{\varepsilon}\left(u_{1}-u_{2}\right)\right)_{\Gamma} \\
& \quad-\beta \int_{\tau}^{t}\left(\Delta_{\Gamma}\left(\eta_{1}-\eta_{2}\right), \operatorname{sign}_{\varepsilon}\left(u_{1}-u_{2}\right)\right)_{\Gamma} \leq 0 .
\end{aligned}
$$

Note that we cannot integrate directly over $(0, t)$ since (5.31) and (5.33) do not extend to $\tau=0$; in other words, we do not have sufficient regularity to use $\operatorname{sign}_{\varepsilon}\left(u_{1}-u_{2}\right)$ as a test function over $(0, T)$. Hence, we first need to take the limit $\varepsilon \searrow 0$, obtaining

$$
\left\|\vartheta_{1}(t)-\vartheta_{2}(t)\right\|_{1}+\alpha\left\|\eta_{1}(t)-\eta_{2}(t)\right\|_{1, \Gamma} \leq\left\|\vartheta_{1}(\tau)-\vartheta_{2}(\tau)\right\|_{1}+\alpha\left\|\eta_{1}(\tau)-\eta_{2}(\tau)\right\|_{1, \Gamma} .
$$

Letting $\tau \searrow 0$ and noting that energy solutions are continuous with values in $L^{1}$ (cf. (2.15)), we obtain the assertion. The proof is concluded.

Remark 5.5. In principle, the above proof does not rely directly on hypothesis (5.30) (or (5.29)). In other words, the conditions on initial data assumed in Theorem 5.1 may suffice. However, in that case the uniqueness statement may be vacuous since we do not know whether there exist energy solutions satisfying (5.31) and (5.33). Actually, even when (5.30) (or (5.29) ) holds, the proved properties does not exclude that there might exist other energy solutions of Problem (P) that do not regularize with respect to time. The same observation can also be referred to the case when $\alpha=0$ and $\beta>0$ since (5.33) is not known to hold under these conditions.

\section{References}

[1] N.D. Alikakos and R. Rostamian, Large time behavior of solutions of Neumann boundary value problem for the porous medium equation, Indiana Univ. Math. J., 30 (1981), 749-785.

[2] A. Blanchet, M. Bonforte, J. Dolbeault, G. Grillo, and J.L. Vázquez, Asymptotics of the fast diffusion equation via entropy estimates, Arch. Rational Mech. Anal., 191 (2009), 347-385. 
[3] G. Bluman and S. Kumei, On the remarkable nonlinear diffusion equation $(\partial / \partial x)[a(u+$ $\left.b)^{-2}(\partial u / \partial x)\right]-(\partial u / \partial t)=0$, J. Math. Phys., 21 (1980), 1019-1023.

[4] M. Bonforte and J.L. Vázquez, Positivity, local smoothing, and Harnack inequalities for very fast diffusion equations, Adv. Math., 223 (2009), 529-578.

[5] M. Bonforte, G. Grillo, and J.L. Vázquez, Special fast diffusion with slow asymptotics: entropy method and flow on a Riemann manifold, Arch. Rational Mech. Anal., 196 (2010), 631-680.

[6] H. Brezis, and W. Strauss, Semi-linear second-order elliptic equations in $L^{1}$, J. Math. Soc. Japan, 25 (1973), 565-590.

[7] F. Brezzi and G. Gilardi, FEM Mathematics, in Finite Element Handbook (H. Kardestuncer Ed.), Part I: Chapt. 1: Functional Analysis, 1.1-1.5; Chapt. 2: Functional Spaces, 2.1-2.11; Chapt. 3: Partial Differential Equations, 3.1-3.6, McGraw-Hill Book Co., New York, 1987.

[8] J.T. Chayes, S.J. Osher, and J.V. Ralston, On singular diffusion equations with applications to self-organized criticality, Comm. Pure Appl. Math., 46 (1993), 1363-1377.

[9] P. Colli, G. Gilardi, E. Rocca, and G. Schimperna, On a Penrose-Fife phase-field model with nonhomogeneous Neumann boundary conditions for the temperature, Differential Integral Equations, 17 (2004), 511-534.

[10] P. Colli and Ph. Laurençot, Weak solutions to the Penrose-Fife phase field model for a class of admissible heat flux laws, Phys. D, 111 (1998), 311-334.

[11] P. Colli, Ph. Laurençot, and J. Sprekels, Global solution to the Penrose-Fife phase field model with special heat flux laws, Variations of domain and free-boundary problems in solid mechanics (Paris, 1997), 181-188, Solid Mech. Appl., 66, Kluwer Acad. Publ., Dordrecht, 1999.

[12] P. Daskalopoulos and M. Del Pino, On nonlinear parabolic equations of very fast diffusion, Arch. Rational Mech. Anal., 137 (1997), 363-380.

[13] P.G. De Gennes, Wetting: statics and dynamics, Reviews of Modern Physics, 57 (1985), 13631377 .

[14] G. Grillo and M. Muratori, Sharp short and long time $L^{\infty}$ bounds for solutions to porous media equations with homogeneous Neumann boundary conditions, J. Differential Equations, 254 (2013), 2261-2288.

[15] M.A. Herrero and M. Pierre, The Cauchy problem for $u_{t}=\Delta u^{m}$ when $0<m<1$, Trans. Amer. Math. Soc., 291 (1985), 145-158.

[16] O. Penrose and P.C. Fife, Thermodynamically consistent models of phase-field type for the kinetics of phase transitions, Phys. D, 43 (1990), 44-62.

[17] G. Schimperna, Global and exponential attractors for the Penrose-Fife system, Math. Models Methods Appl. Sci., 19 (2009), 969-991.

[18] G. Schimperna, A. Segatti, and S. Zelik, Asymptotic uniform boundedness of energy solutions to the Penrose-Fife model, J. Evol. Equ., 12 (2012), 863-890.

[19] J.L. Vázquez, Nonexistence of solutions for nonlinear heat equations of fast-diffusion type, J. Math. Pures Appl. (9), 71 (1992), 503-526.

[20] J.L. Vázquez, "Smoothing and Decay Estimates for Nonlinear Diffusion Equations", Oxford Lecture Series in Mathematics and its Applications, Vol. 33. Oxford University Press, Oxford, 2006.

[21] J.L. Vázquez, "The Porous Medium Equation", Oxford Mathematical Monographs. The Clarendon Press. Oxford University Press, Oxford, 2007. 